\title{
Anisotropic Mesh Adaptation for Crack Propagation Induced by a Thermal Shock
}

\author{
Nicola Ferro ${ }^{a}$, Stefano Micheletti ${ }^{a}$, Simona Perotto $^{a}$ \\ ${ }^{a}$ MOX - Modellistica e Calcolo Scientifico \\ Dipartimento di Matematica \\ Politecnico di Milano \\ Piazza L. da Vinci, 32, I-20133 Milano, Italy \\ \{nicola.ferro, stefano.micheletti, simona.perotto\}@polimi.it
}

February 8, 2017

\begin{abstract}
In this paper we focus on the thermo-mechanical model proposed in $[1,2]$ which describes crack genesis and propagation in brittle materials induced by a thermal shock. Our goal is to provide an efficient numerical technique which employs a computational finite element mesh finely customized to the problem at hand to simulate such phenomena. In particular, we generate automatically adapted anisotropic grids able to closely follow the narrow bands of the damage, driven by a theoretically sound mathematical tool. We carry out two numerical tests to assess the computational performance of the proposed method.
\end{abstract}

Keywords:

crack propagation, brittle materials, anisotropic grids, mesh adaptation, finite elements

\section{Introduction}

We address the numerical simulation of crack propagation induced by a thermal shock in a brittle slab. This issue characterizes, for instance, the shrinkage of materials induced by cooling or drying, which may produce arrays of evenly spaced cracks. Several fields in engineering applications can be affected by such phenomenon, such as thermal shock crack patterns on a glass or ceramic slab [3, 4] or cracking in cement paste matrix upon drying [5].

The capability of accurately predicting the crack path is highly advisable in order to prevent a possible weakness of the material and to preserve the safety of the structure.

Aim of this work is to extend the a posteriori analysis and the numerical procedure proposed in $[6,7,8]$, where both anti-plane and plane linear elasticity models are applied to notched brittle plates, to the initiation and propagation of cracks by a thermal shock in an unnotched material [1, 2]. Actually, models including preexisting cracks are more recurrent in the specialized literature, even though they skip the initiation phase. Both the pre-cracked and the new damage models are based on the Ambrosio-Tortorelli variational formulation in a quasi-static setting, and include irreversibility and energy balance principles $[9,10]$. In particular, the damage is modeled through an auxiliary variable, namely, a phase field, which introduces a form of viscous regularization to 
track the crack path. Recent developments on this subject can be found, e.g., in the special issue on phase field approaches to fracture [11].

To make effective the proposed theoretical analysis and the numerical approach, we enrich a standard finite element discretization with a proper choice of the computational mesh. For this purpose, we employ adapted grids, which distribute in a nonuniform way the mesh elements, so that they are more concentrated close to the crack and coarser where the material is undamaged. Additionaly, we suitably orient and stretch the elements in order to match the intrinsic directional features of the damage $[12,13,14,15,16]$. These meshes are known as anisotropic adapted grids. We provide an automatic tool to generate these customized meshes, moving from a rigorous theoretical analysis, cast in the framework of a posteriori discretization error control (see, e.g., [17]). In [6, 7, 8], we have already assessed the computational advantages led by anisotropic mesh adaptation in terms of computational saving, with respect to both standard (isotropic) adapted and uniform meshes [18]. The a posteriori error analysis in [8] is here extended to the thermo-mechanical process.

The paper is organized as follows. In Section 2, we detail the adopted energy-based mathematical-physical model, formulated in a variational framework as the minimization of an energy functional comprising the elastic and the fracture contributions. Section 3 steps to the discrete counterpart of the variational setting and rigorously derives the anisotropic a posteriori error estimator. In Section 4, the actual algorithm (MACProX = Mesh Adaptation for Crack Propagation with thermal shocKS) assembling the minimization of the functional and the mesh adaptation procedure is set, and numerically investigated in Section 5. Finally, some conclusions are drawn in the last section.

\section{An energy approach to crack modeling}

The physical principle driving crack propagation is represented by Griffith's criterion [19]. This consists of a quasi-static energy-based formulation, which minimizes the total energy of the system, composed by a term modeling the elastic energy of the body, and a fracture term which takes into account the dissipation due to the propagation of the fracture, $\Gamma$. The total energy is analytically represented by

$$
\mathcal{J}_{G}(t ; \mathbf{u}, \Gamma(t))=\int_{\Omega \backslash \Gamma} W(\varepsilon) d \mathbf{x}+W_{\text {ext }}(t, \mathbf{u})+\mathcal{D}(\Gamma(t)),
$$

where $\Omega \subset \mathbb{R}^{d}$, with $d=2,3$, is the volume occupied by the continuum in the reference configuration, $W(\varepsilon)$ is the elastic energy density out of the fracture, $W_{e x t}(t, \mathbf{u})$ is the work of the external forces, $\mathbf{u}$ being the displacement field and $t$ the time, $\mathcal{D}(\Gamma(t))$ represents the dissipation energy released with the propagation of the fracture.

The following result is equivalent to Griffith's criterion [20, 21]:

Proposition 1. The pair $(\Gamma(t), \boldsymbol{u}(t))$ satisfies the equilibrium equation for an elastic continuum and the conditions of Griffith's criterion, if and only if

1. $(\Gamma(t), \boldsymbol{u}(t))$ is a stationary point of $\mathcal{J}_{G}(t ; \boldsymbol{u}, \Gamma)$;

2. $d \Gamma(t) / d t \geq 0$ to guarantee the irreversible evolution of the fracture;

3. the total energy of the system is constant in time. 
In Griffith's original formulation, the dissipation is proportional to the $d$-measure of the fracture. Thus, following $[20,21]$, we replace contribution $\mathcal{D}(\Gamma(t))$ in $(1)$ with the Haussdorf measure of $\Gamma(t)$, which yields the new functional

$$
\mathcal{J}_{H}(t ; \mathbf{u}, \Gamma(t))=\int_{\Omega \backslash \Gamma} W(\varepsilon) d \mathbf{x}+W_{\text {ext }}(t, \mathbf{u})+G_{c} \mathcal{H}^{d-1}(\Gamma(t)),
$$

where $\mathcal{H}^{d-1}(\Gamma)$ is the Haussdorf measure of $\Gamma$, and $G_{c}$ is the so-called body toughness.

Nevertheless, the minimization of $\mathcal{J}_{H}$ is quite unpractical, at least in order to perform numerical simulations due to the different geometric dimensionality of the terms involved in (2), and to the evolutive nature of $\Gamma(t)$. This justifies the introduction in the literature of Griffithlike energy functionals, simpler to deal with from both an analytical and a computational viewpoint [22, 23, 24, 25]. In particular, following [1, 2], the energy-like functional employed in this work is based on the one proposed by L. Ambrosio and V.M. Tortorelli [9], enriched with a thermal contribution, with a view to modeling the thermal shock effect. The temperature evolution due to the shock is described by the heat equation for the temperature field $T=T(\mathbf{x}, t)$,

$$
\begin{cases}\frac{\partial T}{\partial t}-\nabla \cdot\left(k_{c} \nabla T\right)=0 & \text { in } \Omega \times\left(0, t_{F}\right] \\ T=T_{0}-\Delta T & \text { on } \gamma_{\text {shock }} \times\left(0, t_{F}\right] \\ T(\mathbf{x}, 0)=T_{0} & \text { on } \Omega,\end{cases}
$$

with $T_{0}$ the initial temperature (before cooling down the body), $t_{F}$ the final time, $k_{c}$ the thermal diffusivity, $\Delta T$ the temperature drop applied to the boundary portion $\gamma_{\text {shock }}$.

The coupled thermo-mechanical model is based on the minimization of the functional

$$
\mathcal{J}(\mathbf{u}, \alpha)=\frac{1}{2} \int_{\Omega}(1-\alpha)^{2} A\left(\varepsilon(\mathbf{u})-\varepsilon_{t h}\right):\left(\varepsilon(\mathbf{u})-\varepsilon_{t h}\right) d \mathbf{x}+\int_{\Omega} \frac{G_{c}}{4 c_{w}}\left(\frac{\alpha}{l}+l|\nabla \alpha|^{2}\right) d \mathbf{x},
$$

where $\varepsilon(\mathbf{u})=1 / 2\left(\nabla \mathbf{u}+(\nabla \mathbf{u})^{T}\right)$ is the total strain tensor, $c_{w}=\int_{0}^{1} \sqrt{s} d s$ is a normalization constant, $A$ is the stiffness tensor, $\varepsilon_{t h}=\varepsilon_{t h}(T)=\mu\left(T_{0}-T\right) \mathbf{I}$ is the inelastic strain due to a thermal shock, $\mu$ being the thermal volumetric expansion coefficient, $T_{0}-T$ the temperature drop and $\mathbf{I}$ the identity tensor, and $\alpha: \Omega \rightarrow[0,1]$ is the phase field tracking the crack, with $\alpha=0$ where the material is perfectly sound, $\alpha=1$ where the material is fully damaged. In practice, the phase field exhibits a sharp variation on a spatial scale on the order of $l$, which represents the so-called internal length. Notice that functional $\mathcal{J}$ involves only the pure elastic strain, $\varepsilon(\mathbf{u})-\varepsilon_{t h}$.

In addition to the constraint $0 \leq \alpha \leq 1$, we still have to enforce the physical irreversibility of crack propagation, i.e., no healing is allowed. This leads to add a further constraint on $\alpha$, namely, $\alpha(t) \geq \alpha(\bar{t})$ for any $t \geq \bar{t}, \bar{t}$ being any time in $\left(0, t_{F}\right)$.

Finally, we emphasize that model (3)-(4), in contrast to Griffith's model, does not necessarily require a pre-existing notch in the domain, the thermal shock being responsible for both crack initiation and propagation $[1,2]$. In more detail, we may distinguish two main phases separated by a characteristic time $t^{*}$, such that, for $t \leq t^{*}$, the damage field $\alpha$ is uniformly distributed along the boudary $\gamma_{\text {shock }}$, where it exhibits a sharp gradient; vice versa, for $t>t^{*}$, the damage field bifurcates and band-like cracks form and propagate.

\subsection{The minimization of the functional}

We observe that the energy functional $\mathcal{J}$ in (4) is not convex with respect to the pair $(\mathbf{u}, \alpha)$, even though it is convex with respect to each variable, separately. This will represent an issue with a view to the numerical optimization algorithm. 
Before dealing with the minimization of $\mathcal{J}$, we provide the following result, whose proof is straightforward:

Proposition 2. Functional $\mathcal{J}$ is Gâteaux differentiable in $S=\left[H^{1}(\Omega)\right]^{d} \times\left[H^{1}(\Omega) \cap L^{\infty}(\Omega)\right]$. In particular, the Gâteaux derivative of $\mathcal{J}$ at $(\boldsymbol{u}, \alpha) \in S$ in the direction $(\boldsymbol{v}, \beta) \in S$ is

$$
\begin{aligned}
\mathcal{J}^{\prime}(\boldsymbol{u}, \alpha ; \boldsymbol{v}, \beta)= & \underbrace{\int_{\Omega}(1-\alpha)^{2} A\left(\varepsilon(\boldsymbol{u})-\varepsilon_{t h}\right): \varepsilon(\boldsymbol{v}) d \mathbf{x}}_{a(\alpha ; \boldsymbol{u}, \boldsymbol{v})}+ \\
& \underbrace{\int_{\Omega}\left[(\alpha-1) \beta A\left(\varepsilon(\boldsymbol{u})-\varepsilon_{t h}\right):\left(\varepsilon(\boldsymbol{u})-\varepsilon_{t h}\right)+\frac{c \beta}{l}+2 c l \nabla \alpha \cdot \nabla \beta\right] d \mathbf{x}}_{b(\boldsymbol{u} ; \alpha, \beta)}
\end{aligned}
$$

with $c=G_{c} /\left(4 c_{w}\right)$.

For numerical purposes, we deal with a time discrete formulation based on the partition $0=t_{0}<t_{1}<\ldots<t_{N}=t_{F}$, consisting of $N$ uniform intervals. Then, we introduce the function spaces $U=\left[H^{1}(\Omega)\right]^{d}$ and $W_{k}=\left\{w \in H^{1}(\Omega): \chi \leq w\left(t_{k}\right) \leq 1\right.$ a.e. in $\left.\Omega\right\}$, for a given function $\chi \in H^{1}(\Omega ;[0,1])$. To enforce the monotonicity condition associated with the irreversibility, function $\chi$ will be always chosen as $w\left(t_{k-1}\right)$.

Thus, the minimization of functional $\mathcal{J}$ constrained by irreversibility can be stated as: Find the pair $(\mathbf{u}, \alpha) \in U \times W_{k}$ at time $t_{k}$, for $k=1, \ldots, N$, such that

$$
(\mathbf{u}, \alpha) \in \operatorname{argmin}\left\{\mathcal{T}\left(\mathbf{u}^{*}, \alpha^{*}\right): \mathbf{u}^{*} \in U ; \alpha^{*} \in W_{k}\right\} .
$$

Equivalently, problem (5) can be recast as: Find the pair $(\mathbf{u}, \alpha) \in U \times W_{k}$ at time $t_{k}$, for $k=1, \ldots, N$, such that

$$
\left\{\begin{array}{l}
a(\alpha ; \mathbf{u}, \mathbf{v})=0 \quad \forall \mathbf{v} \in U \\
b(\mathbf{u} ; \alpha, \alpha-\beta) \leq 0 \quad \forall \beta \in W_{k}
\end{array}\right.
$$

$\left[26,2\right.$, 27]. The pair $(\mathbf{u}, \alpha) \in U \times W_{k}$ is a critical point of $\mathcal{J}$ if system (6) is satisfied.

Remark 1. If we replace the variational inequality in (6) by the correspoding equality constraint $b(\boldsymbol{u} ; \alpha, \beta)=0$ for any $\beta \in H^{1}(\Omega)$, we can prove a maximum principle on the phase field $\alpha$, i.e., $\alpha \leq 1$. For this purpose, we apply the change of variable $z=1-\alpha$ in $b(\boldsymbol{u} ; \alpha, \beta)=0$. This yields a reaction-diffusion equation for $z$ with a constant positive diffusion coefficient, $2 c l$, a non-negative reaction term, $A\left(\varepsilon(\boldsymbol{u})-\varepsilon_{t h}\right):\left(\varepsilon(\boldsymbol{u})-\varepsilon_{t h}\right)$, and a positive source term, $c / l$, which implies $z \geq 0$, i.e., $\alpha \leq 1$. Notice that, the lower bound $\alpha \geq 0$ cannot be guaranteed, a priori, due to the linear term $\alpha / l$ in the definition of $\mathcal{J}$.

\section{Anisotropic error analysis}

With a view to the numerical simulation, first we discretize problems (3) and (6) via a classical finite element scheme; then, we derive an a posteriori error estimator in an anisotropic setting to drive the mesh adaptation procedure. Hereafter, we assume $d=2$. 


\subsection{The discrete setting}

Let $\left\{\mathcal{T}_{h}\right\}$ be a family of conformal meshes of $\bar{\Omega}$, with $h>0$ the characteristic mesh size of $\mathcal{T}_{h}$ [28]. We associate with $\mathcal{T}_{h}=\{K\}$ the space $X_{h}$ of the continuous piecewise linear finite elements, with $K$ the generic element of the triangulation $\mathcal{T}_{h}$.

We first consider the discretization of the mechanical problem. The discrete counterpart of the energy functional $\mathcal{J}(\mathbf{u}, \alpha)$ is provided by

$$
\mathcal{J}_{h}\left(\mathbf{u}_{h}, \alpha_{h}\right)=\frac{1}{2} \int_{\Omega} P_{h}\left(\left(1-\alpha_{h}\right)^{2}\right) A\left(\varepsilon\left(\mathbf{u}_{h}\right)-\varepsilon_{t h}\right):\left(\varepsilon\left(\mathbf{u}_{h}\right)-\varepsilon_{t h}\right) d \mathbf{x}+\int_{\Omega} \frac{G_{c}}{4 c_{w}}\left(\frac{\alpha_{h}}{l}+l\left|\nabla \alpha_{h}\right|^{2}\right) d \mathbf{x},
$$

where $\mathbf{u}_{h} \in U_{h}=\left[X_{h}\right]^{2}$ and $\alpha_{h} \in X_{h}$ approximate the displacement and the phase field, respectively while $P_{h}: C^{0}(\bar{\Omega}) \rightarrow X_{h}$ is the Lagrange interpolant onto the space $X_{h}$ [28].

Analogously to the continuous case, we can compute the Gâteaux derivative of $\mathcal{J}_{h}$ at $\left(\mathbf{u}_{h}, \alpha_{h}\right) \in$ $S_{h}=U_{h} \times X_{h}$ in the direction $\left(\mathbf{v}_{h}, \beta_{h}\right) \in S_{h}$, given by $\mathcal{J}_{h}^{\prime}\left(\mathbf{u}_{h}, \alpha_{h} ; \mathbf{v}_{h}, \beta_{h}\right)=a_{h}\left(\alpha_{h} ; \mathbf{u}_{h}, \mathbf{v}_{h}\right)+$ $b_{h}\left(\mathbf{u}_{h} ; \alpha_{h}, \beta_{h}\right)$, with

$$
\begin{gathered}
a_{h}\left(\alpha_{h} ; \mathbf{u}_{h}, \mathbf{v}_{h}\right)=\int_{\Omega} P_{h}\left(\left(1-\alpha_{h}\right)^{2}\right) A\left(\varepsilon\left(\mathbf{u}_{h}\right)-\varepsilon_{t h}\right): \varepsilon\left(\mathbf{v}_{h}\right) d \mathbf{x}, \\
b_{h}\left(\mathbf{u}_{h} ; \alpha_{h}, \beta_{h}\right)=\int_{\Omega}\left(P_{h}\left(\left(\alpha_{h}-1\right)\right) \beta_{h} A\left(\varepsilon\left(\mathbf{u}_{h}\right)-\varepsilon_{t h}\right):\left(\varepsilon\left(\mathbf{u}_{h}\right)-\varepsilon_{t h}\right)+\frac{c \beta_{h}}{l}+2 c l \nabla \alpha_{h} \cdot \nabla \beta_{h}\right) d \mathbf{x} .
\end{gathered}
$$

Thus, the discrete counterpart of (5) is: Find the pair $\left(\mathbf{u}_{h}, \alpha_{h}\right) \in U_{h} \times W_{k}^{h}$ at time $t_{k}$, for $k=1, \ldots, N$, such that

$$
\left(\mathbf{u}_{h}, \alpha_{h}\right) \in \operatorname{argmin}\left\{\mathcal{J}_{h}\left(\mathbf{u}_{h}^{*}, \alpha_{h}^{*}\right): \mathbf{u}_{h}^{*} \in U_{h} ; \alpha_{h}^{*} \in W_{k}^{h}\right\},
$$

with $W_{k}^{h}=\left\{w_{h} \in X_{h}: \chi_{h} \leq w_{h}\left(t_{k}\right) \leq 1\right.$ a.e. in $\left.\Omega\right\}, \chi_{h}$ being a finite element approximation to $\chi$.

Problem (8) is actually a box-constrained optimization problem, i.e., we are led to solve the coupled differential system

$$
\begin{cases}a_{h}\left(\alpha_{h} ; \mathbf{u}_{h}, \mathbf{v}_{h}\right)=0 & \forall \mathbf{v}_{h} \in U_{h} \\ b_{h}\left(\mathbf{u}_{h} ; \alpha_{h}, \alpha_{h}-\beta_{h}\right) \leq 0 & \forall \beta_{h} \in W_{k}^{h} .\end{cases}
$$

Nevertheless, for theoretical purposes, we consider only the case where the damage field $\alpha_{h}$ is an interior point of the box, namely, we deal with the two coupled equations

$$
\left\{\begin{array}{lll}
a_{h}\left(\alpha_{h} ; \mathbf{u}_{h}, \mathbf{v}_{h}\right)=0 & \forall \mathbf{v}_{h} \in U_{h} \\
b_{h}\left(\mathbf{u}_{h} ; \alpha_{h}, \beta_{h}\right)=0 & \forall \beta_{h} \in X_{h} .
\end{array}\right.
$$

However, as addressed in Section 5, we actually include the irreversibility constraint in the numerical algorithm.

Remark 2. Analogously to Remark 1 , a discrete maximum principle holds also for $\alpha_{h}$, i.e., $\alpha_{h} \leq 1$, under the condition $k_{i j}=\int_{\Omega} \nabla \xi_{i} \cdot \nabla \xi_{j} d \mathbf{x} \leq 0$ for any $i \neq j$, where $\xi_{k}$ is the $k$-th basis (hat) function of space $X_{h}$ (see also [18, 6]). Indeed, equation $(9)_{2}$ amounts to a discretized diffusionreaction equation for the variable $z_{h}=1-\alpha_{h}$, with a constant positive diffusion coefficient, $2 c l$, a non-negative reaction term, $A\left(\varepsilon\left(\boldsymbol{u}_{h}\right)-\varepsilon_{t h}\right):\left(\varepsilon\left(\boldsymbol{u}_{h}\right)-\varepsilon_{t h}\right)$ weighting a diagonal mass matrix lumped via the $P_{h}$ operator, and a positive source term, $c / l$. The condition on $k_{i j}$ is the standard constraint for the stiffness matrix to satisfy a discrete maximum principle [29].

Concerning the discretization of the heat equation (3), we still adopt the finite element space $X_{h}$ to discretize the spatial dependence, while we resort to a backward Euler scheme for time advancing. 


\subsection{The anisotropic setting}

We refer to the setting used in $[6,7,8]$, according to which the anisotropic source of information is provided by the affine map $T_{K}: \hat{K} \rightarrow K$ between the reference triangle $\hat{K}$ and the generic triangle $K \in \mathcal{T}_{h}$, such that

$$
\mathbf{x}=\left[x_{1}, x_{2}\right]^{T}=T_{K}(\hat{\mathbf{x}})=M_{K} \hat{\mathbf{x}}+\mathbf{b}_{K} \quad \forall \hat{\mathbf{x}} \in \hat{K},
$$

where $M_{K} \in \mathbb{R}^{2 \times 2}$ is a matrix which deforms $\hat{K}$ and $\mathbf{b}_{K} \in \mathbb{R}^{2}$ performs a shift. In particular, we select as a reference element $\hat{K}$ the equilateral triangle inscribed in the unit circle centered at the origin. Map $T_{K}$ allows us to describe the geometric features of any triangle $K \in \mathcal{T}_{h}$ by exploiting the spectral properties of the Jacobian $M_{K}$. For this purpose, we first introduce the polar decomposition $M_{K}=B_{K} Z_{K}$ of $M_{K}$, with $B_{K}, Z_{K} \in \mathbb{R}^{2 \times 2}$ a symmetric positive definite and an orthogonal matrix, respectively; then, we compute the eigenvalue/eigenvector decomposition of $B_{K}, B_{K}=R_{K}^{T} \Lambda_{K} R_{K}$, where $\Lambda_{K}=\operatorname{diag}\left(\lambda_{1, K}, \lambda_{2, K}\right)$ with $\lambda_{1, K} \geq \lambda_{2, K}$ the eigenvalues of $B_{K}$, and $R_{K}^{T}=\left[\mathbf{r}_{1, K}, \mathbf{r}_{2, K}\right]$ the column matrix of the unit eigenvectors of $B_{K}$. Geometrically, $\left\{\lambda_{i, K}, \mathbf{r}_{i, K}\right\}, i=$ 1,2 , provide the half-length and the direction of the axes of the ellipse circumscribed to $K$, which is the image of the unit circle through $T_{K}$ (see Figure 1). The aspect ratio $s_{K}=\lambda_{1, K} / \lambda_{2, K} \geq 1$ measures the deformation of $K$, being $s_{K}=1$ for equilateral triangles.

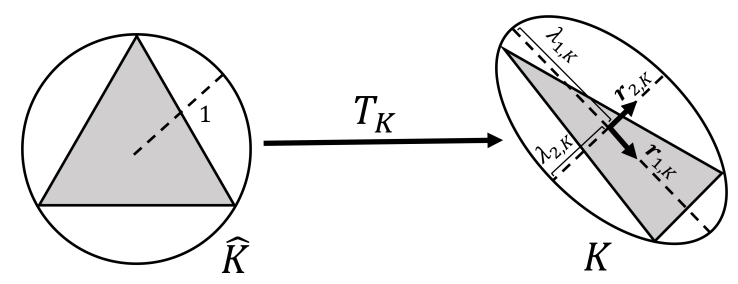

Figure 1: Map $T_{K}$ changes the reference triangle $\hat{K}$ into the generic one $K$.

With a view to the a posteriori analysis, we introduce some anisotropic error estimates for the quasi-interpolant Clément operator, $C_{h}: L^{2}(\Omega) \rightarrow X_{h}[30]$.

Lemma 1. Let $w \in H^{1}(\Omega)$. If $\operatorname{card}\left(\mathcal{T}_{h}\right) \leq \mathcal{N} \in \mathbb{N}^{+}$, and $\operatorname{diam}\left(T_{K}^{-1}\left(\Delta_{K}\right)\right) \leq C_{\Delta} \simeq \operatorname{diam}(\hat{K})$, then there exist constants $C_{s}=C_{s}\left(\mathcal{N}, C_{\Delta}\right)$, with $s=1,2,3$, such that, for any $K \in \mathcal{T}_{h}$, it holds

$$
\begin{gathered}
\left\|w-C_{h}(w)\right\|_{L^{2}(K)} \leq C_{1}\left[\sum_{i=1}^{2} \lambda_{i, K}^{2}\left(\boldsymbol{r}_{i, K}^{T} G_{\Delta_{K}}(w) \boldsymbol{r}_{i, K}\right)\right]^{\frac{1}{2}}, \\
\left|w-C_{h}(w)\right|_{H^{1}(K)} \leq C_{2} \frac{1}{\lambda_{2, K}}\left[\sum_{i=1}^{2} \lambda_{i, K}^{2}\left(\boldsymbol{r}_{i, K}^{T} G_{\Delta_{K}}(w) \boldsymbol{r}_{i, K}\right)\right]^{\frac{1}{2}}, \\
\left\|w-C_{h}(w)\right\|_{L^{2}(\partial K)} \leq C_{3}\left(\frac{h_{K}}{\lambda_{1, K} \lambda_{2, K}}\right)^{\frac{1}{2}}\left[\sum_{i=1}^{2} \lambda_{i, K}^{2}\left(\boldsymbol{r}_{i, K}^{T} G_{\Delta_{K}}(w) \boldsymbol{r}_{i, K}\right)\right]^{\frac{1}{2}},
\end{gathered}
$$

where $\Delta_{K}=\left\{T \in \mathcal{T}_{h}: T \cap K \neq \emptyset\right\}$ is the patch of the elements associated with $K, h_{K}=\operatorname{diam}(K)$, 
$G_{\Delta_{K}}$ is the symmetric positive semi-definite matrix given by

$$
G_{\Delta_{K}}(w)=\left[\begin{array}{ll}
\int_{\Delta_{K}}\left(\frac{\partial w}{\partial x_{1}}\right)^{2} d \boldsymbol{x} & \int_{\Delta_{K}} \frac{\partial w}{\partial x_{1}} \frac{\partial w}{\partial x_{2}} d \boldsymbol{x} \\
\int_{\Delta_{K}} \frac{\partial w}{\partial x_{1}} \frac{\partial w}{\partial x_{2}} d \boldsymbol{x} & \int_{\Delta_{K}}\left(\frac{\partial w}{\partial x_{2}}\right)^{2} d \boldsymbol{x}
\end{array}\right] .
$$

For the proof of these estimates we refer to [31, 32].

We finally recall an equivalence result between the standard $H^{1}\left(\Delta_{K}\right)$-seminorm and its anisotropic counterpart.

Lemma 2. Let $w \in H^{1}(\Omega)$, and $K \in \mathcal{T}_{h}$. For any $\gamma_{1}, \gamma_{2}>0$, it holds that

$$
\min \left(\gamma_{1}, \gamma_{2}\right) \leq \frac{\gamma_{1}\left(\boldsymbol{r}_{1, K}^{T} G_{\Delta_{K}}(w) \boldsymbol{r}_{1, K}\right)+\gamma_{2}\left(\boldsymbol{r}_{2, K}^{T} G_{\Delta_{K}}(w) \boldsymbol{r}_{2, K}\right)}{|w|_{H^{1}\left(\Delta_{K}\right)}^{2}} \leq \max \left(\gamma_{1}, \gamma_{2}\right) .
$$

For the proof we refer to [13].

\subsection{The anisotropic a posteriori error estimator}

The main result of this work is provided by the following

Proposition 3. Let $\left(\boldsymbol{u}_{h}, \alpha_{h}\right)$ be a solution to (9). Then, for any $(\boldsymbol{v}, \beta) \in\left[H^{1}(\Omega)\right]^{2} \times H^{1}(\Omega)$, we have

$$
\left|\mathcal{J}^{\prime}\left(\boldsymbol{u}_{h}, \alpha_{h} ; \boldsymbol{v}, \beta\right)\right| \leq \widetilde{C} \sum_{K \in \mathcal{T}_{h}}\left(\sum_{i=1}^{2}\left[\rho_{i, K}^{A}\left(\alpha_{h}, \boldsymbol{u}_{h}\right) \omega_{K}\left(v_{i}\right)\right]+\rho_{K}^{B}\left(\boldsymbol{u}_{h}, \alpha_{h}\right) \omega_{K}(\beta)\right)
$$

with $\widetilde{C}=\widetilde{C}\left(\mathcal{N}, C_{\Delta}\right)$ constant,

$$
\begin{gathered}
\rho_{i, K}^{A}\left(\alpha_{h}, \boldsymbol{u}_{h}\right)=\left\|2\left(1-\alpha_{h}\right) \sigma_{i}^{t h}\left(\boldsymbol{u}_{h}\right) \cdot \nabla \alpha_{h}\right\|_{L^{2}(K)}+\frac{1}{2}\left\|\llbracket \sigma_{i}^{t h}\left(\boldsymbol{u}_{h}\right) \rrbracket\right\|_{L^{\infty}(\partial K)}\left\|\left(1-\alpha_{h}\right)^{2}\right\|_{L^{2}(\partial K)}\left(\frac{h_{K}}{\lambda_{1, K} \lambda_{2, K}}\right)^{\frac{1}{2}} \\
+\left\|\left(1-\alpha_{h}\right)^{2}-P_{h}\left(\left(1-\alpha_{h}\right)^{2}\right)\right\|_{L^{\infty}(K)}\left\|\sigma_{i}^{t h}\left(\boldsymbol{u}_{h}\right)\right\|_{L^{2}(K)} \frac{1}{\lambda_{2, K}} \\
\rho_{K}^{B}\left(\boldsymbol{u}_{h}, \alpha_{h}\right)=\left\|\left(\alpha_{h}-1\right) \sigma^{t h}\left(\boldsymbol{u}_{h}\right):\left(\varepsilon\left(\boldsymbol{u}_{h}\right)-\varepsilon_{t h}\right)+\frac{c}{l}\right\|_{L^{2}(K)}+c l\left\|\llbracket \nabla \alpha_{h} \rrbracket\right\|_{L^{2}(\partial K)}\left(\frac{h_{K}}{\lambda_{1, K} \lambda_{2, K}}\right)^{\frac{1}{2}} \\
+\left\|\sigma^{t h}\left(\boldsymbol{u}_{h}\right):\left(\varepsilon\left(\boldsymbol{u}_{h}\right)-\varepsilon_{t h}\right)\right\|_{L^{2}(K)} h_{K}^{2}\left|\alpha_{h}\right|_{W^{1, \infty}(K)} \frac{1}{\lambda_{2, K}}, \\
\omega_{K}(\xi)=\left[\sum_{i=1}^{2} \lambda_{i, K}^{2}\left(\boldsymbol{r}_{i, K}^{T} G_{\Delta_{K}}(\xi) \boldsymbol{r}_{i, K}\right)\right]^{\frac{1}{2}}, \quad \forall \xi \in H^{1}(\Omega),
\end{gathered}
$$

where

$$
\llbracket \sigma_{i}^{t h}\left(\boldsymbol{u}_{h}\right) \rrbracket= \begin{cases}{\left[\sigma_{i}^{t h}\left(\boldsymbol{u}_{h}\right) \cdot \boldsymbol{n}\right]_{e}} & e \in \mathcal{E}_{h} \cap \Omega, \\ \left.2 \sigma_{i}^{t h}\left(\boldsymbol{u}_{h}\right) \cdot \boldsymbol{n}\right|_{e} & e \in \mathcal{E}_{h} \cap \partial \Omega\end{cases}
$$


and

$$
\llbracket \nabla \alpha_{h} \rrbracket= \begin{cases}{\left[\nabla \alpha_{h} \cdot \boldsymbol{n}\right]_{e}} & e \in \mathcal{E}_{h} \cap \Omega, \\ \left.2 \nabla \alpha_{h} \cdot \boldsymbol{n}\right|_{e} & e \in \mathcal{E}_{h} \cap \partial \Omega\end{cases}
$$

denote the generalized jump of the $i$-th component, $\sigma_{i}^{\text {th }}\left(\boldsymbol{u}_{h}\right)$, of the normal thermal stress tensor $\sigma^{\text {th }}\left(\boldsymbol{u}_{h}\right)=A\left(\varepsilon\left(\boldsymbol{u}_{h}\right)-\varepsilon_{t h}\right)$ and of the normal derivative of $\alpha_{h}$, respectively, $[\cdot]_{e}$ is the standard jump across the edge e $\in \mathcal{E}_{h} \cap \Omega, \mathcal{E}_{h}$ is the skeleton of $\mathcal{T}_{h}, \boldsymbol{n}$ is the unit outward normal vector to $e$.

Proof. Exploiting Proposition 2 with $(\mathbf{u}, \alpha)=\left(\mathbf{u}_{h}, \alpha_{h}\right)$, we have

$$
\left|\mathcal{T}^{\prime}\left(\mathbf{u}_{h}, \alpha_{h} ; \mathbf{v}, \beta\right)\right| \leq\left|a\left(\alpha_{h} ; \mathbf{u}_{h}, \mathbf{v}\right)\right|+\left|b\left(\mathbf{u}_{h} ; \alpha_{h}, \beta\right)\right| .
$$

We first address term $\left|a\left(\alpha_{h} ; \mathbf{u}_{h}, \mathbf{v}\right)\right|$. Thanks to (9), we have, for any $\mathbf{v}_{h} \in\left[X_{h}\right]^{2}$

$$
\left|a\left(\alpha_{h} ; \mathbf{u}_{h}, \mathbf{v}\right)\right| \leq\left|a\left(\alpha_{h} ; \mathbf{u}_{h}, \mathbf{v}-\mathbf{v}_{h}\right)\right|+\left|a\left(\alpha_{h} ; \mathbf{u}_{h}, \mathbf{v}_{h}\right)-a_{h}\left(\alpha_{h} ; \mathbf{u}_{h}, \mathbf{v}_{h}\right)\right| .
$$

We start by considering the first contribution on the right-hand side. Integrating by parts, we have

$$
\begin{aligned}
& a\left(\alpha_{h} ; \mathbf{u}_{h}, \mathbf{v}-\mathbf{v}_{h}\right)=\sum_{K \in \mathcal{T}_{h}}\left\{\int_{K}\left(1-\alpha_{h}\right)^{2} \sigma^{t h}\left(\mathbf{u}_{h}\right): \varepsilon\left(\mathbf{v}-\mathbf{v}_{h}\right) d \mathbf{x}\right\} \\
= & \sum_{K \in \mathcal{T}_{h}}\left\{\int_{K} 2\left(1-\alpha_{h}\right) \nabla \alpha_{h} \cdot \sigma^{t h}\left(\mathbf{u}_{h}\right)\left(\mathbf{v}-\mathbf{v}_{h}\right) d \mathbf{x}+\int_{\partial K}\left(1-\alpha_{h}\right)^{2} \sigma^{t h}\left(\mathbf{u}_{h}\right)\left(\mathbf{v}-\mathbf{v}_{h}\right) \cdot \mathbf{n} d s\right\} .
\end{aligned}
$$

To keep the directional information, we introduce the componentwise representation

$$
a\left(\alpha_{h} ; \mathbf{u}_{h}, \mathbf{v}-\mathbf{v}_{h}\right)=\sum_{i=1}^{2} a_{i}\left(\alpha_{h} ; \mathbf{u}_{h}, v_{i}-v_{h, i}\right),
$$

with $\mathbf{v}=\left[v_{1}, v_{2}\right]^{T}, \mathbf{v}_{h}=\left[v_{h, 1}, v_{h, 2}\right]^{T}$, and

$$
\begin{aligned}
a_{i}\left(\alpha_{h} ; \mathbf{u}_{h}, v_{i}-v_{h, i}\right) & =\sum_{K \in \mathcal{T}_{h}}\left\{\int_{K} 2\left(1-\alpha_{h}\right) \nabla \alpha_{h} \cdot \sigma_{i}^{t h}\left(\mathbf{u}_{h}\right)\left(v_{i}-v_{h, i}\right) d \mathbf{x}\right. \\
& \left.+\int_{\partial K}\left(1-\alpha_{h}\right)^{2} \sigma_{i}^{t h}\left(\mathbf{u}_{h}\right) \cdot \mathbf{n}\left(v_{i}-v_{h, i}\right) d s\right\} .
\end{aligned}
$$

Thanks to Hölder and Cauchy-Schwarz inequalities and definition (11), we have, for $i=1,2$,

$$
\begin{aligned}
\left|a_{i}\left(\alpha_{h} ; \mathbf{u}_{h}, v_{i}-v_{h, i}\right)\right| & \leq \sum_{K \in \mathcal{T}_{h}}\left\{\left\|2\left(1-\alpha_{h}\right) \sigma_{i}^{t h}\left(\mathbf{u}_{h}\right) \cdot \nabla \alpha_{h}\right\|_{L^{2}(K)}\left\|v_{i}-v_{h, i}\right\|_{L^{2}(K)}\right. \\
& \left.+\frac{1}{2}\left\|\llbracket \sigma_{i}^{t h}\left(\mathbf{u}_{h}\right) \rrbracket\right\|_{L^{\infty}(\partial K)}\left\|\left(1-\alpha_{h}\right)^{2}\right\|_{L^{2}(\partial K)}\left\|v_{i}-v_{h, i}\right\|_{L^{2}(\partial K)}\right\} .
\end{aligned}
$$

We choose $v_{h, i}=C_{h}\left(v_{i}\right)$ and use Lemma 1 , to obtain

$$
\begin{aligned}
\left|a_{i}\left(\alpha_{h} ; \mathbf{u}_{h}, \mathbf{v}-\mathbf{v}_{h}\right)\right| & \leq C_{4} \sum_{K \in \mathcal{T}_{h}}\left\{\left\|2\left(1-\alpha_{h}\right) \sigma_{i}^{t h}\left(\mathbf{u}_{h}\right) \cdot \nabla \alpha_{h}\right\|_{L^{2}(K)}\right. \\
& \left.+\frac{1}{2}\left\|\llbracket \sigma_{i}^{t h}\left(\mathbf{u}_{h}\right) \rrbracket\right\|_{L^{\infty}(\partial K)}\left\|\left(1-\alpha_{h}\right)^{2}\right\|_{L^{2}(\partial K)}\left(\frac{h_{K}}{\lambda_{1, K} \lambda_{2, K}}\right)^{\frac{1}{2}}\right\} \omega_{K}\left(v_{i}\right),
\end{aligned}
$$


with $C_{4}=\max \left(C_{1}, C_{3}\right)$.

Concerning the second contribution on the right-hand side of (13), we have

$$
a\left(\alpha_{h} ; \mathbf{u}_{h}, \mathbf{v}_{h}\right)-a_{h}\left(\alpha_{h} ; \mathbf{u}_{h}, \mathbf{v}_{h}\right)=\int_{\Omega}\left(\left(1-\alpha_{h}\right)^{2}-P_{h}\left(\left(1-\alpha_{h}\right)^{2}\right)\right) \sigma^{t h}\left(\mathbf{u}_{h}\right): \varepsilon\left(\mathbf{v}_{h}\right) d \mathbf{x} .
$$

Splitting this term componentwise, passing to a summation over the mesh elements, and using Hölder and Cauchy-Schwarz inequalities, we get

$$
\begin{aligned}
& \left|a_{i}\left(\alpha_{h} ; \mathbf{u}_{h}, \mathbf{v}_{h}\right)-a_{h, i}\left(\alpha_{h} ; \mathbf{u}_{h}, \mathbf{v}_{h}\right)\right| \\
& \leq \sum_{K \in \mathcal{T}_{h}}\left\{\left\|\left(1-\alpha_{h}\right)^{2}-P_{h}\left(\left(1-\alpha_{h}\right)^{2}\right)\right\|_{L^{\infty}(K)}\left\|\sigma_{i}^{t h}\left(\mathbf{u}_{h}\right)\right\|_{L^{2}(K)}\left(\left\|\nabla v_{h, i}-\nabla v_{i}\right\|_{L^{2}(K)}+\left\|\nabla v_{i}\right\|_{L^{2}(K)}\right)\right\} .
\end{aligned}
$$

Picking $v_{h, i}=C_{h}\left(v_{i}\right)$, and exploiting Lemmas 1 and 2 , with $\gamma_{1}=\lambda_{1, K}^{2}, \gamma_{2}=\lambda_{2, K}^{2}$, we have

$$
\begin{aligned}
& \left|a_{i}\left(\alpha_{h} ; \mathbf{u}_{h}, \mathbf{v}_{h}\right)-a_{h, i}\left(\alpha_{h} ; \mathbf{u}_{h}, \mathbf{v}_{h}\right)\right| \\
& \leq\left(1+C_{2}\right) \sum_{K \in \mathcal{T}_{h}}\left\{\left\|\left(1-\alpha_{h}\right)^{2}-P_{h}\left(\left(1-\alpha_{h}\right)^{2}\right)\right\|_{L^{\infty}(K)}\left\|\sigma_{i}^{t h}\left(\mathbf{u}_{h}\right)\right\|_{L^{2}(K)} \frac{1}{\lambda_{2, K}} \omega_{K}\left(v_{i}\right)\right\} .
\end{aligned}
$$

By collecting the estimates (14) and (15), we obtain the following estimate for the first term in (12).

$$
\left|a\left(\alpha_{h} ; \mathbf{u}_{h}, \mathbf{v}\right)\right| \leq C_{5} \sum_{K \in \mathcal{T}_{h}} \sum_{i=1}^{2} \rho_{i, K}^{A}\left(\alpha_{h}, \mathbf{u}_{h}\right) \omega_{K}\left(v_{i}\right),
$$

with $C_{5}=\max \left(C_{4}, 1+C_{2}\right)$.

Now we address the second term in (12). Using (9), for any $\beta_{h} \in X_{h}$, we have

$$
\left|b\left(\mathbf{u}_{h} ; \alpha_{h}, \beta\right)\right| \leq\left|b\left(\mathbf{u}_{h} ; \alpha_{h}, \beta-\beta_{h}\right)\right|+\left|b\left(\mathbf{u}_{h} ; \alpha_{h}, \beta_{h}\right)-b_{h}\left(\mathbf{u}_{h} ; \alpha_{h}, \beta_{h}\right)\right|,
$$

and we consider the two terms on the right-hand side, separately. For the first one, by combining integration by parts with Cauchy-Schwarz inequality, it follows that

$$
\begin{aligned}
& \left|b\left(\mathbf{u}_{h} ; \alpha_{h}, \beta-\beta_{h}\right)\right|= \\
& =\left|\int_{\Omega}\left[\left(\alpha_{h}-1\right)\left(\beta-\beta_{h}\right) \sigma^{t h}\left(\mathbf{u}_{h}\right):\left(\varepsilon\left(\mathbf{u}_{h}\right)-\varepsilon_{t h}^{h}\right)+\frac{c}{l}\left(\beta-\beta_{h}\right)+2 c l \nabla \alpha_{h} \cdot \nabla\left(\beta-\beta_{h}\right)\right] d \mathbf{x}\right| \\
& \leq \sum_{K \in \mathcal{T}_{h}}\left|\int_{K}\left(\left(\alpha_{h}-1\right) \sigma^{t h}\left(\mathbf{u}_{h}\right):\left(\varepsilon\left(\mathbf{u}_{h}\right)-\varepsilon_{t h}^{h}\right)+\frac{c}{l}\right)\left(\beta-\beta_{h}\right) d \mathbf{x}+2 c l \int_{\partial K}\left(\beta-\beta_{h}\right) \nabla \alpha_{h} \cdot \mathbf{n} d s\right| \\
& \leq \sum_{K \in \mathcal{T}_{h}}\left\{\left\|\left(\alpha_{h}-1\right) \sigma^{t h}\left(\mathbf{u}_{h}\right):\left(\varepsilon\left(\mathbf{u}_{h}\right)-\varepsilon_{t h}^{h}\right)+\frac{c}{l}\right\|_{L^{2}(K)}\left\|\beta-\beta_{h}\right\|_{L^{2}(K)}+c l\left\|\llbracket \nabla \alpha_{h} \rrbracket\right\|\left\|_{L^{2}(\partial K)}\right\| \beta-\beta_{h} \|_{L^{2}(\partial K)}\right\} .
\end{aligned}
$$

Now, we identify $\beta_{h}$ with $C_{h}(\beta)$ and we exploit Lemma 1 to obtain

$$
\begin{aligned}
& \left|b\left(\mathbf{u}_{h} ; \alpha_{h}, \beta-\beta_{h}\right)\right| \leq \\
& \leq C_{4} \sum_{K \in \mathcal{T}_{h}}\left[\left\|\left(\alpha_{h}-1\right) \sigma^{t h}\left(\mathbf{u}_{h}\right):\left(\varepsilon\left(\mathbf{u}_{h}\right)-\varepsilon_{t h}^{h}\right)+\frac{c}{l}\right\|_{L^{2}(K)}+c l\left\|\llbracket \nabla \alpha_{h} \rrbracket\right\|_{L^{2}(\partial K)}\left(\frac{h_{K}}{\lambda_{1, K} \lambda_{2, K}}\right)^{\frac{1}{2}}\right] \omega_{K}(\beta) .
\end{aligned}
$$


For the second term on the right-hand side of (17), we apply successively Cauchy-Schwarz inequality, the standard isotropic estimate for the $L^{2}$-norm of the interpolation error associated with $P_{h}$, and the auxiliary result $\left|w_{h} \varphi_{h}\right|_{H^{2}(K)} \leq 2\left|w_{h}\right|_{W^{1, \infty}(K)}\left\|\nabla \varphi_{h}\right\|_{L^{2}(K)}$ for any $w_{h}, \varphi_{h} \in X_{h}$ and for any $K \in \mathcal{T}_{h}$, to have

$$
\begin{aligned}
& \left|b\left(\mathbf{u}_{h}, \alpha_{h}, \beta_{h}\right)-b_{h}\left(\mathbf{u}_{h}, \alpha_{h}, \beta_{h}\right)\right| \leq \sum_{K \in \mathcal{T}_{h}}\left\|\alpha_{h} \beta_{h}-P_{h}\left(\alpha_{h} \beta_{h}\right)\right\|_{L^{2}(K)}\left\|\sigma^{t h}\left(\mathbf{u}_{h}\right):\left(\varepsilon\left(\mathbf{u}_{h}\right)-\varepsilon_{t h}^{h}\right)\right\|_{L^{2}(K)} \\
& \leq C_{6} \sum_{K \in \mathcal{T}_{h}}\left\|\sigma^{t h}\left(\mathbf{u}_{h}\right):\left(\varepsilon\left(\mathbf{u}_{h}\right)-\varepsilon_{t h}^{h}\right)\right\|_{L^{2}(K)} h_{K}^{2}\left|\alpha_{h} \beta_{h}\right|_{H^{2}(K)} \\
& \leq 2 C_{6} \sum_{K \in \mathcal{T}_{h}}\left\|\sigma^{t h}\left(\mathbf{u}_{h}\right):\left(\varepsilon\left(\mathbf{u}_{h}\right)-\varepsilon_{t h}^{h}\right)\right\|_{L^{2}(K)} h_{K}^{2}\left|\alpha_{h}\right|_{W^{1, \infty}(K)}\left\|\nabla \beta_{h}\right\|_{L^{2}(K)} \\
& \leq 2 C_{6}\left(1+C_{2}\right) \sum_{K \in \mathcal{T}_{h}}\left\|\sigma^{t h}\left(\mathbf{u}_{h}\right):\left(\varepsilon\left(\mathbf{u}_{h}\right)-\varepsilon_{t h}^{h}\right)\right\|_{L^{2}(K)} h_{K}^{2}\left|\alpha_{h}\right|_{W^{1, \infty}(K)} \frac{1}{\lambda_{2, K}} \omega_{K}(\beta)
\end{aligned}
$$

after adding and subtracting $\beta$ in the last norm of the third line, and combining Lemma 1 and Lemma 2 with $\gamma_{1}=\lambda_{1, K}^{2}$ and $\gamma_{2}=\lambda_{2, K}^{2}$, and where $C_{6}$ is the constant of the interpolation error estimate in the $L^{2}$-norm for $P_{h}$. Gathering (18) and (19) provides the final estimate

$$
\left|b\left(\mathbf{u}_{h} ; \alpha_{h}, \beta\right)\right| \leq C_{7} \sum_{K \in \mathcal{T}_{h}} \rho_{K}^{B}\left(\mathbf{u}_{h}, \alpha_{h}\right) \omega_{K}(\beta),
$$

with $C_{7}=\max \left(C_{4}, 2 C_{6}\left(1+C_{2}\right)\right)$.

The thesis (10) easily follows, recalling (12) and using (16) and (20), with $\widetilde{C}=\max \left(C_{5}, C_{7}\right)$.

In order to define a computable a posteriori error estimator, exploiting the arbitrariness of $\mathbf{v}$ and $\beta$, we choose $\mathbf{v}=\mathbf{u}-\mathbf{u}_{h}$ and $\beta=\alpha-\alpha_{h}$. This leads us to identify the global error estimator with

$$
\eta=\sum_{K \in \mathcal{T}_{h}} \eta_{K}\left(\mathbf{u}_{h}, \alpha_{h}\right)
$$

where the local error estimator is given by

$$
\eta_{K}\left(\mathbf{u}_{h}, \alpha_{h}\right)=\sum_{i=1}^{2}\left[\rho_{i, K}^{A}\left(\alpha_{h}, \mathbf{u}_{h}\right) \omega_{K}^{R}\left(u_{i}-u_{h, i}\right)\right]+\rho_{K}^{B}\left(\mathbf{u}_{h}, \alpha_{h}\right) \omega_{K}^{R}\left(\alpha-\alpha_{h}\right),
$$

where

$$
\omega_{K}^{R}\left(w-w_{h}\right)=\left[\sum_{i=1}^{2} \lambda_{i, K}^{2}\left(\mathbf{r}_{i, K}^{T} G_{\Delta_{K}}^{R}\left(w-w_{h}\right) \mathbf{r}_{i, K}\right)\right]^{\frac{1}{2}}, \quad \text { for } \quad w=u_{i}, \alpha,
$$

with $G_{\Delta_{K}}^{R}\left(w-w_{h}\right)$ the matrix whose generic entry is

$$
\left[G_{\Delta_{K}}^{R}\left(w-w_{h}\right)\right]_{i j}=\int_{\Delta_{K}}\left(w_{i}^{Z Z}-\frac{\partial w_{h}}{\partial x_{i}}\right)\left(w_{j}^{Z Z}-\frac{\partial w_{h}}{\partial x_{j}}\right) d \mathbf{x},
$$

where $\mathbf{w}^{Z Z}=\left[w_{1}^{Z Z}, w_{2}^{Z Z}\right]^{T}$ is the well-known Zienkiewicz-Zhu recovered gradient [33, 34].

The choice made above for $\mathbf{v}$ and $\beta$ can be justified on a more rigorous mathematical ground. Indeed, according to Corollary 3.4 in [6], $\eta$ estimates, up to a third-order remainder $R$, the energy error, since it holds that

$$
\mathcal{J}(\mathbf{u}, \alpha)-\mathcal{J}\left(\mathbf{u}_{h}, \alpha_{h}\right)=\frac{1}{2} \mathcal{J}^{\prime}\left(\mathbf{u}_{h}, \alpha_{h} ; \mathbf{u}-\mathbf{u}_{h}, \alpha-\alpha_{h}\right)+R .
$$




\section{The numerical procedure}

We employ a metric-based mesh adaptation procedure driven by estimator $\eta$. In particular, moving from the anisotropic information contained in the local estimators $\eta_{K}$, we derive a piecewise constant metric field, $\widetilde{M}_{K}=\widetilde{R}_{K}^{T} \widetilde{\Lambda}_{K}^{-2} \widetilde{R}_{K}$, with $\widetilde{R}_{K}^{T}=\left[\widetilde{\mathbf{r}}_{1, K}, \tilde{\mathbf{r}}_{2, K}\right]$ and $\widetilde{\Lambda}_{K}=\operatorname{diag}\left(\tilde{\lambda}_{1, K}, \tilde{\lambda}_{2, K}\right)$, on the actual mesh and then we use it to build the new adapted mesh. In particular, the metric is obtained by enforcing an error equidistribution criterion combined with the minimization of the number of mesh elements under a constraint on the magnitude of $\eta$.

According to [6], we summarize the information identifying the new metric $\widetilde{M}_{K}$ in the following

Proposition 4. Let $\left\{\boldsymbol{t}_{i, K}, g_{i, K}\right\}$ with $i=1,2$, be the eigenvector/eigenvalue pairs of

$$
\Gamma_{K}=\sum_{i=1}^{2}\left[\bar{\rho}_{i, K}^{A}\left(\alpha_{h}, \boldsymbol{u}_{h}\right)\right]^{2} \bar{G}_{\Delta_{K}}^{R}\left(u_{i}-u_{h, i}\right)+\left[\bar{\rho}_{K}^{B}\left(\boldsymbol{u}_{h}, \alpha_{h}\right)\right]^{2} \bar{G}_{\Delta_{K}}^{R}\left(\alpha-\alpha_{h}\right),
$$

with $g_{1, K} \geq g_{2, K}>0$, and

$$
\bar{\rho}_{i, K}^{A}\left(\alpha_{h}, \mathbf{u}_{h}\right)=\frac{\rho_{i, K}^{A}\left(\alpha_{h}, \mathbf{u}_{h}\right)}{\left(|\hat{K}| \lambda_{1, K} \lambda_{2, K}\right)^{\frac{1}{2}}}, \quad \bar{\rho}_{K}^{B}\left(\mathbf{u}_{h}, \alpha_{h}\right)=\frac{\rho_{K}^{B}\left(\boldsymbol{u}_{h}, \alpha_{h}\right)}{\left(|\hat{K}| \lambda_{1, K} \lambda_{2, K}\right)^{\frac{1}{2}}}, \quad \bar{G}_{\Delta_{K}}^{R}(\cdot)=\frac{G_{\Delta_{K}}^{R}(\cdot)}{\left(|\hat{K}| \lambda_{1, K} \lambda_{2, K}\right)},
$$

where $|\hat{K}|$ is the area of the reference element, and $\lambda_{i, K}, i=1,2$, are defined as in Section 3.2 on the actual mesh. Then, the components of the metric $\widetilde{M}_{K}$ are

$$
\tilde{\mathbf{r}}_{1, K}=\boldsymbol{t}_{2, K}, \quad \lambda_{1, K}=\left(\frac{1}{|\hat{K}| \sqrt{2}}\left(\frac{g_{1, K}}{g_{2, K}^{2}}\right)^{\frac{1}{2}} \frac{E T O L}{\# \mathcal{T}_{h}}\right)^{\frac{1}{3}}, \quad \lambda_{2, K}=\left(\frac{1}{|\hat{K}| \sqrt{2}}\left(\frac{g_{2, K}}{g_{1, K}^{2}}\right)^{\frac{1}{2}} \frac{E T O L}{\# \mathcal{T}_{h}}\right)^{\frac{1}{3}}
$$

where ETOL is the accuracy required on the estimator $\eta$, and $\# \mathcal{T}_{h}$ denotes the cardinality of the actual mesh $\mathcal{T}_{h}$.

For more details about the derivation of this result, we refer, e.g., to [12, 13, 14, 15, 16]. Metric $\widetilde{M}_{K}$ is finally provided as an input to a metric-based mesh adaptation tool to build the new adapted mesh. In the numerical assessment below, we employ the bidimensional anisotropic mesh generator of FreeFem++ [35].

We now merge this mesh adaptation procedure with the minimization of the functional $\mathcal{J}_{h}$ in (7). This miminization is not a trivial task since the functional is not convex, whereas it is strictly convex with respect to each variable, separately.

In practice, we commute from mesh adaptation to minimization of $\mathcal{J}_{h}$, until both the mesh and the functional stagnate to within a given threshold, MTOL $\ll 1$ and FPTOL $\ll 1$, respectively. The minimization phase relies on the alternate minimization algorithm proposed in [36], which we have already employed as a backbone in $[6,7,8]$ where it has been extended to deal with mesh adaptation. Given an initial mesh, $\mathcal{T}_{h}^{(0)}$, the global algorithm is listed below. 


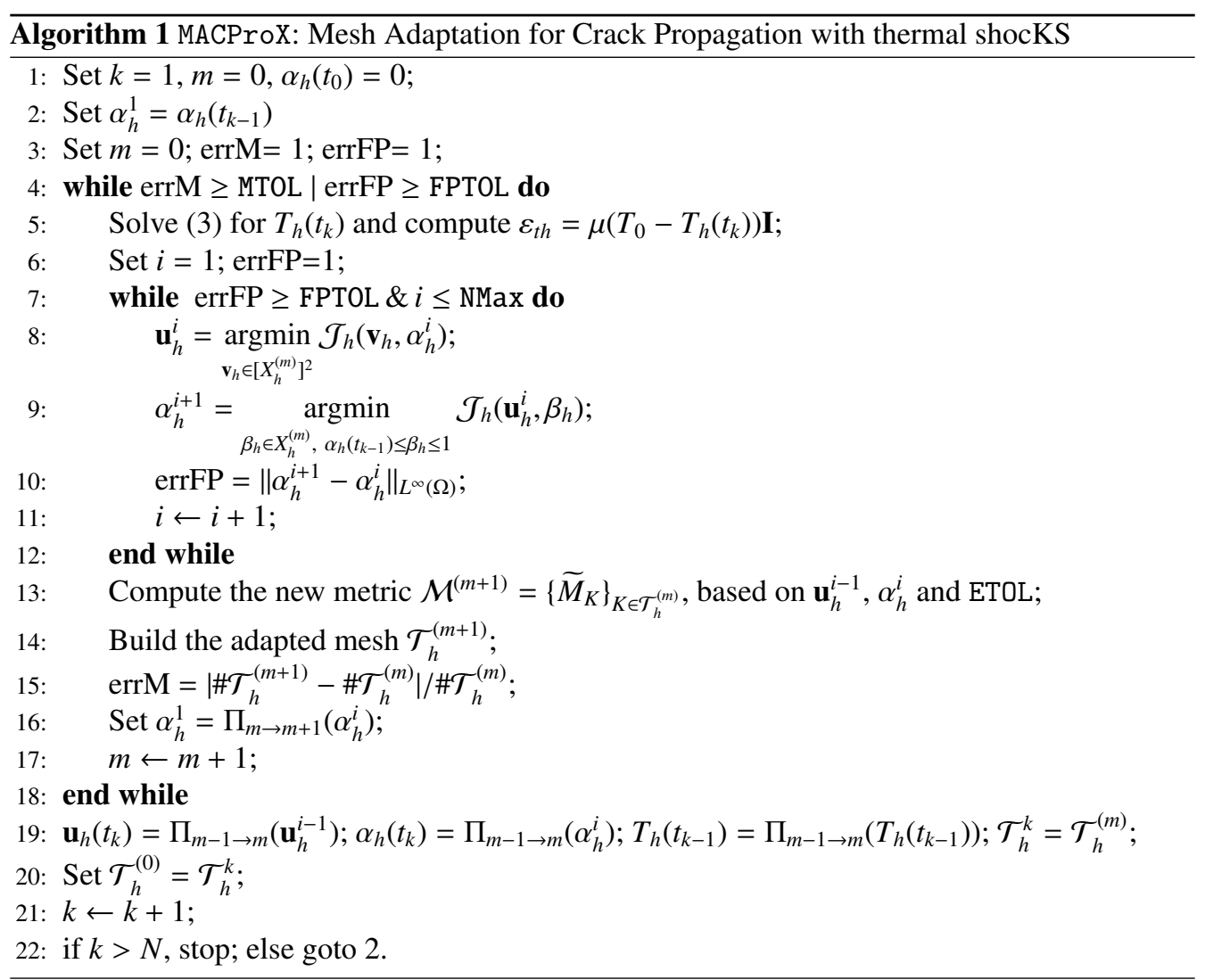

Some comments are in order. The minimization of the functional with respect to $\mathbf{u}_{h}$ and $\alpha_{h}$ is performed by solving the corresponding Euler-Lagrange equations $(9)_{1}$ for $\mathbf{u}_{h}$, namely, a standard linear elliptic problem, whereas for $\alpha_{h}$ we employ the function IPOPT, based on the interior point method, and which deals with a general constrained minimization problem [37]. This allows us to explicitly enforce the irreversibility constraint, which has been omitted in the $a$ posteriori analysis.

A bound, NMax, on the maximum number of minimization steps is fixed before starting the mesh adaptation phase.

Superscripts in the mesh, say $\mathcal{T}_{h}^{(m)}$, designate successive meshes during the adaptation loop, with associated finite element space $X_{h}^{(m)}$. Operator $\Pi_{n \rightarrow n+1}(\cdot)$ denotes the interpolant mapping the finite element functions defined on $\mathcal{T}_{h}^{(n)}$ onto the adapted mesh $\mathcal{T}_{h}^{(n+1)}$, before restarting any new optimization or time loop.

Finally, the convergence of the mesh adaptivity is assessed by checking the relative variation of the number of elements. 


\section{Verification of MACProX algorithm}

We apply algorithm MACProX to two geometric configurations ${ }^{1}$, a square slab and a quarter of a simplified disc brake. In both cases, the specimen is homogeneous, free at the boundary, non-prestressed in its initial configuration, and uniformly heated. Then, it is quenched in a cold bath, which induces a thermal shock along the immersed surface. According to [2], three main phases characterize the evolution of the phenomenon: during the very early stages, a uniform strip propagates orthogonally to the immersed surface; then, at some critical time, a bifurcation occurs and the damage exhibits periodically distributed bands of equal length and grows at the center of these bands; later, a selective crack arrest takes place, where some bands stop while the others keep on propagating.

A square slab. The specimen is the square slab $\Omega=(0,50)[\mathrm{mm}] \times(0,50)[\mathrm{mm}]$, with $\gamma_{\text {shock }}=$ $(0,50) \times\{0\}[\mathrm{mm}]$. The parameters involved in the thermo-mechanical model are gathered in Table 1, where $E, v$ are the Young modulus and the Poisson ratio, respectively, characterizing the stiffness tensor $A$ of a linear elastic homogeneous material. The initial mesh $\mathcal{T}_{h}^{(0)}$ is a uniform

\begin{tabular}{ll} 
Model parameters & \\
\hline$k_{c}$ & $1\left[\mathrm{~mm}^{2} / \mathrm{s}\right]$ \\
$t_{F}$ & $10[\mathrm{~s}]$ \\
$T_{0}$ & $0\left[{ }^{\circ} \mathrm{C}\right]$ \\
$\Delta T$ & $380\left[{ }^{\circ} \mathrm{C}\right]$ \\
$\mu$ & $1.7 \cdot 10^{-3}\left[\mathrm{~K}^{-1}\right]$ \\
$E$ & $340[\mathrm{GPa}]$ \\
$v$ & 0.22 \\
$G_{c}$ & $42.47\left[\mathrm{~J} / \mathrm{m}^{2}\right]$ \\
$c_{w}$ & $2 / 3$ \\
$l$ & $10^{-6}[\mathrm{~m}]$ \\
$N$ & 50
\end{tabular}

Table 1: Parameters for the square slab and the quarter of a disc brake test cases.

unstructured grid consisting of 7400 triangles. The parameters fed to algorithm MACProX are ETOL $=10^{-1}$, MTOL $=10^{-2}$, NMax $=50$ and FPTOL $=10^{-3}$.

Figure 2, left, shows the phase field $\alpha_{h}$ computed on the anisotropic adapted mesh at the final time $t=t_{F}$. On the right, three enlarged views of the bands at time step 10, 30 and 50 (top-bottom). In particular, the contour plot at the top corresponds to the second phase of the evolution, while the other two are associated with the last phase. Colors range from blue (undamaged material) to red (fully damaged slab). In Figure 5, two bird's-eye views of the phase field along with the adapted mesh at the final time are provided.

Figure 3 shows the temperature profile computed at $x_{1}=25[\mathrm{~mm}]$ as a function of $x_{2}$, for the time steps 5, 15 and 45 . It clearly emphasizes the strong boundary layer occurring at the bottom edge where the shock develops. Qualitatively it is similar to Figure 3 in [4].

Figure 4, left, shows the output of the anisotropic mesh adaptation procedure at time step 30 (top) along with three successive zooms in on the bands. The anisotropic feature of the mesh

\footnotetext{
${ }^{1}$ The experiments have been performed using Freefem++ 3.36 64-bit on a Lenovo Ideapad Z510, equipped with a $2.20 \mathrm{GHz}$ Intel Core i7 processor, and $8 \mathrm{~GB}$ of RAM.
} 

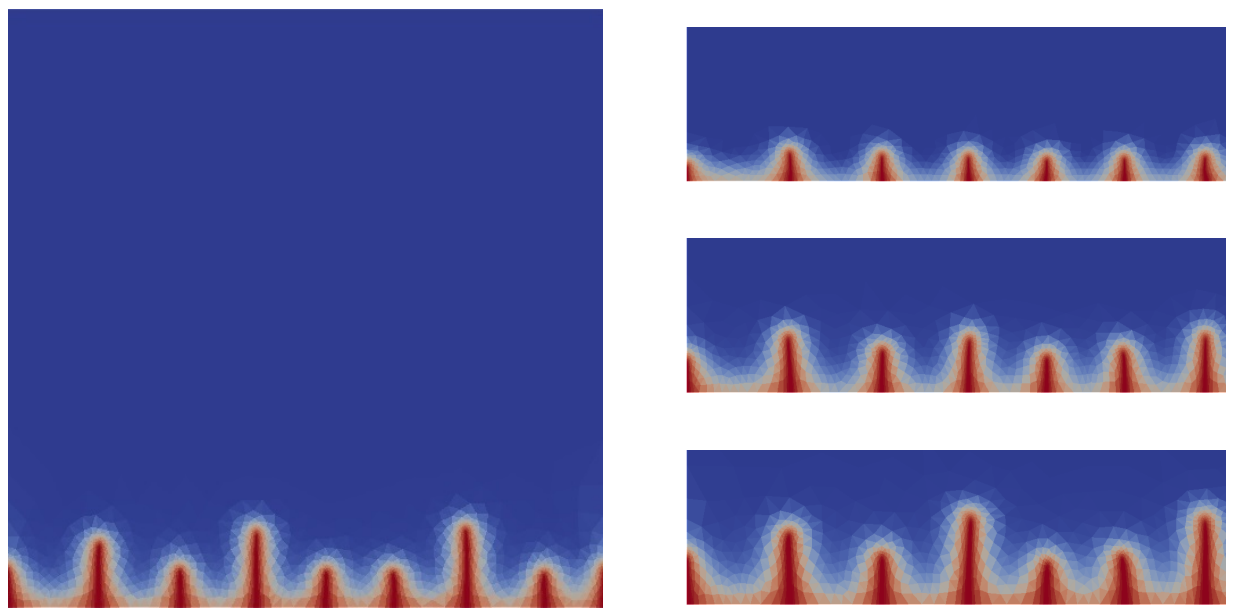

Figure 2: Square slab: phase field at $t=t_{F}$ (left) and enlarged views at time steps 10, 30 and 50 (right, top-bottom)

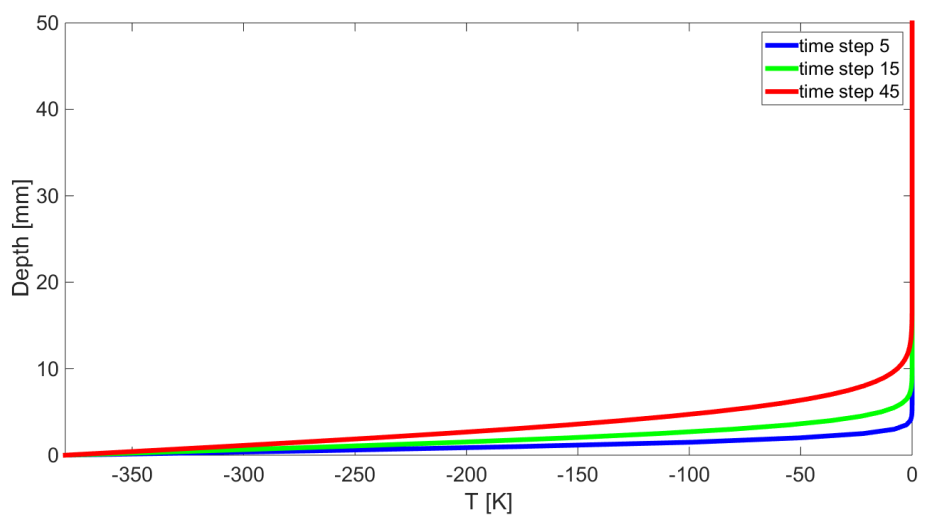

Figure 3: Square slab: temperature profile at $x_{1}=25[\mathrm{~mm}]$ as a function of depth for three different time steps.

is evident. In particular, the maximum aspect ratio over the elements of this mesh is 933. This figure emphasizes the strong anisotropy of the mesh along the crack path, whereas the triangles closer to the tip are still rather isotropic. This should ensure that the crack advancing is not affected by the directionality of the elements. A comparison with an isotropic mesh adaptation is performed to check the advantages of the anisotropic counterpart. In particular, the isotropic metric is obtained by enforcing $s_{K}=1$, for any $K \in \mathcal{T}_{h}$, in Proposition 4, i.e., $\widetilde{M}_{K}=\left(1 / \lambda_{K}^{2}\right) I$, with $I \in \mathbb{R}^{2 \times 2}$ the identity matrix and

$$
\lambda_{K}=\left(\frac{1}{|\hat{K}| \sqrt{2}}\left(\frac{2}{g_{1, K}+g_{2, K}}\right)^{\frac{1}{2}} \frac{\mathrm{ETOL}}{\# \mathcal{T}_{h}}\right)^{\frac{1}{3}} .
$$



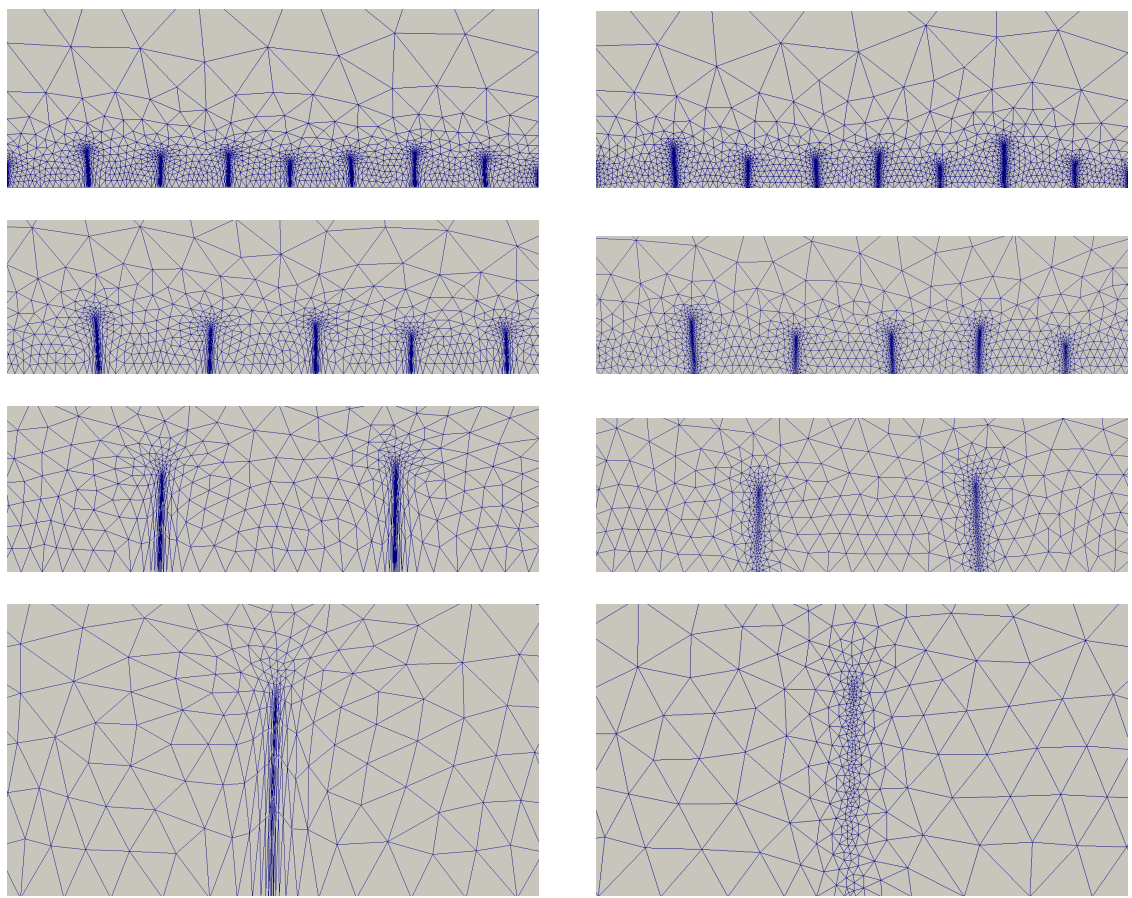

Figure 4: Square lab: anisotropic (left) vs isotropic (right) mesh adaptation at time step 30, for increasing magnification (top-bottom).
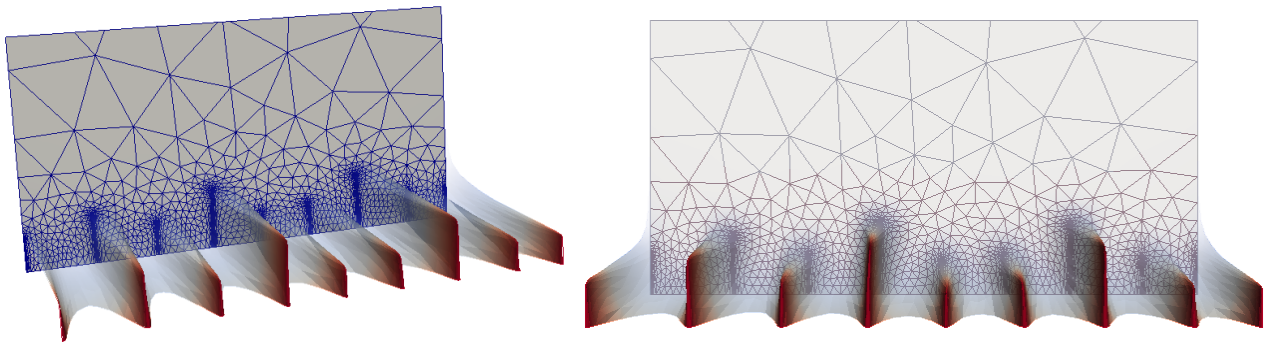

Figure 5: Square slab: 3D views of the phase field overlapped to the adapted mesh at time $t=t_{F}$.

Figure 4 contrasts the two adaptive procedures, highliting the poor tracking of the bands in the case of the isotropic approach. In Figure 6, left, we compare the evolution of the number of elements for the isotropic and anisotropic mesh adaptation. The reduction provided by the anisotropic procedure is remarkable for the whole time span, with greater extent during the last steps. On average, about half the elements are required by the anisotropic meshes. Moreover, in Figure 7, we compare the performance of the two approaches, in terms of total and average (total time/\# loops) time spent in the optimization phase, in correspondence with the last six time steps. In particular, the large discrepancy in terms of the average time can be ascribed to the con- 

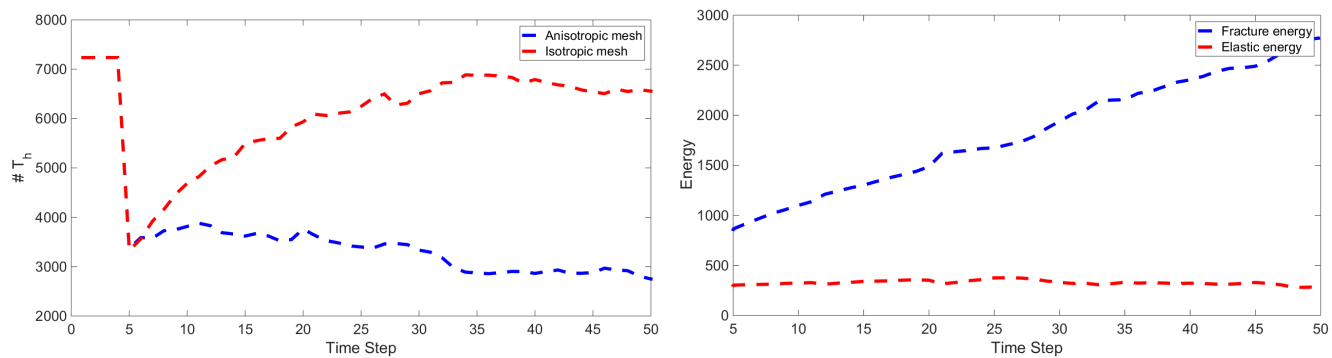

Figure 6: Square slab: evolution of the mesh cardinality (left) and of the energy contributions (right).
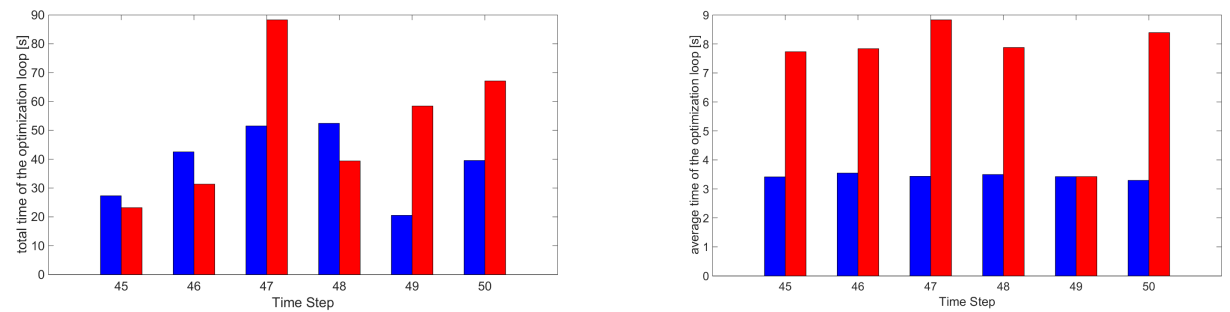

Figure 7: Square slab: total and average time for the optimization loop at the last six time steps, for the anisotropic (left bars) and isotropic (right bars) procedures.
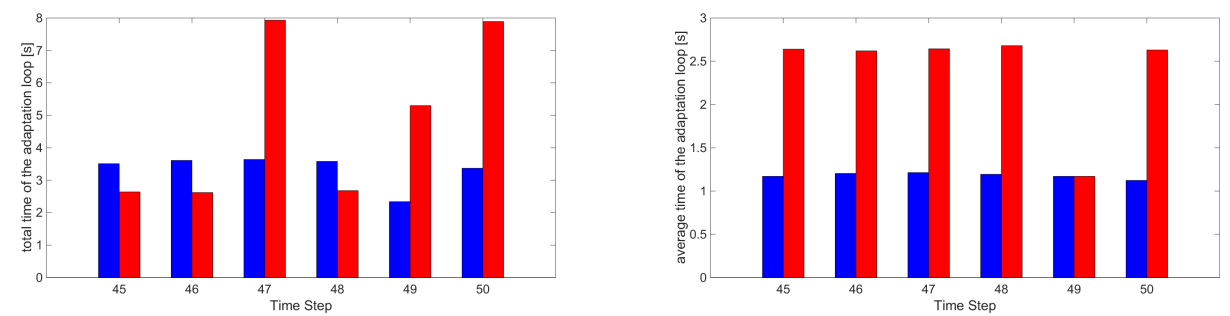

Figure 8: Square slab: total and average time for the adaptation at the last six time steps, for the anisotropic (left bars) and isotropic (right bars) procedures.

siderably lower number of elements in the anisotropic grid. The total time are somewhat more comparable, even though, when anisotropy outperforms, it is remarkably less costly. We carry out the same comparison by considering just the adaptation phase. The total and average time per iteration in corresponence with the last six time steps is shown in Figure 8, left and right, respectively. As for the optimization loop, the anisotropic tool cuts down drastically the time when it improves the isotropic procedure. Overall, the most expensive phase from a computational viewpoint is optimization, which generally demands a time one order higher than adaptation. This is confirmed by the values in Table 2, which show a total computational gain of about $23 \%$ when employing an anisotropic approach. 


\begin{tabular}{cccc}
\hline \hline & total time [s] & optimization time [s] & adaptation time [s] \\
\hline Anisotropic & 4434.91 & 4070.00 & 364.91 \\
Isotropic & 5754.69 & 5223.80 & 530.89 \\
\hline
\end{tabular}

Table 2: Square slab: total, optimization, and adaptation time for the anisotropic and isotropic mesh adaptation.

To conclude, in Figure 6, right, we plot the trend of the two components, elastic and fracture, of the energy functional in (7). The total energy is constantly increasing, and the dominant contribution is due to the crack propagation.

A disc brake. In this second test case, we consider a more interesting configuration, consisting of a quarter of a vehicle disc brake. Actually, these brakes are subject to high thermal stresses during routine braking, with a strong increase while braking hard. The disc brake is modeled as an annular region surrounded by an outer and an inner circular boundary of radius 50 [mm] and $5[\mathrm{~mm}]$, respectively, which identify $\gamma_{\text {shock }}$. To apply the thermo-mechanical model (4), we suppose that crack genesis is stirred by two thermal shocks at both the curved boundaries. The parameters are the same as in Table 1.

The initial mesh $\mathcal{T}_{h}^{(0)}$ is a uniform unstructured grid consisting of 5234 elements. We use the same parameters for algorithm MACProX as in the previous test. Figure 9 shows the elevated
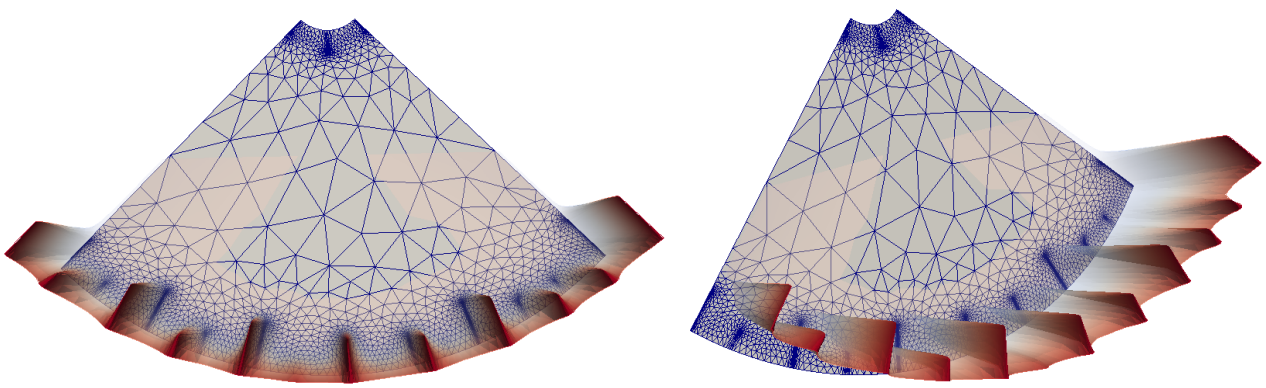

Figure 9: Disc brake: 3D views of the phase field overlapped to the adapted mesh at time $t=t_{F}$.

view from two perspectives of the phase field $\alpha_{h}$ and of the corresponding anisotropic adapted mesh at $t=t_{F}$. In Figure 10, we provide the contour plot of the phase field at the final time, left, and at the four intermediate time levels, $t=5,15,25$, and 45, right. Notice that two groups of cracks arise and propagate inwards, with the expected alternate pattern of propagating and stopped bands. Figure 11 compares the final adapted meshes returned by algorithm MACProX, in the anisotropic, left, and isotropic, right, setting. Macroscopically, the two grids are very similar, since the anisotropic features are localized in the bands. Nevertheless, the anisotropic algorithm detects an additional crack on the outer boundary at the very early stage of its formation. Three successive zooms in on the south-east quadrant are gathered in Figure 12, in the anisotropic, top, and isotropic, bottom, case. These highlights allow us to appreciate the very strong anisotropy inside the bands, the maximum aspect ratio over the elements being equal to 505.35. The advantages brought by the anisotropic approach are emphasized by the plot in Figure 13, left, which shows the evolution of the cardinality of the mesh, in the anisotropic and isotropic cases. 

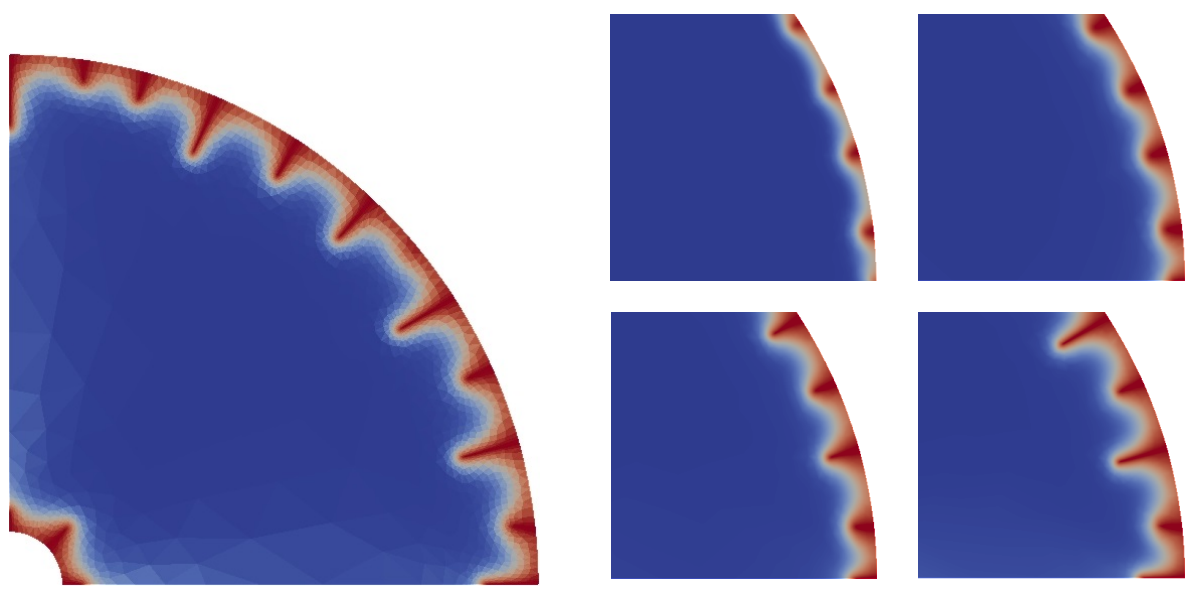

Figure 10: Disc brake: phase field at $t=t_{F}$ (left) and enlarged views at time steps 5, 15, 25, and 45 (right, top-bottom, left-right).
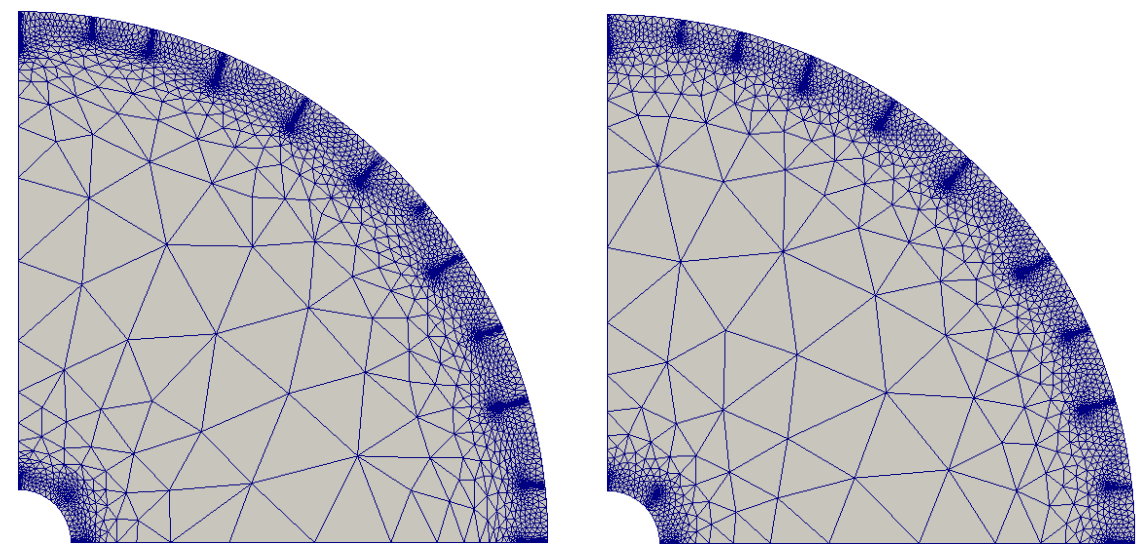

Figure 11: Disc brake: anisotropic (left) vs isotropic (right) mesh adaptation at time step 30.

As for the square slab configuration, the maximum gain is reached quite early, where the isotropic trend exhibits a peak, and in the final steps. At $t=t_{F}$, a reduction of about $32 \%$ is obtained. In Figures 14-15, we perform the same analysis as in Figures 7-8 to check the computational improvement led by the anisotropic procedure in terms of total and average time required for the optimization and the adaptation phases, by focusing on the last six time steps. The gain is more evident in this test case. Globally, the total times are much favorable for the anisotropic setting, whereas a less striking discrepancy characterizes the average times of the adaptation. In particular, the brake configuration is more complex to simulate (see Table 3) as it turns out by the high percentage of adaptation to total time. This ratio is about $23 \%$ and $27 \%$ for the anisotropic and isotropic case, respectively, to be compared with $8 \%$ and $9 \%$ in the square slab specimen. Finally, in Figure 13, right, we plot the time evolution of the elastic and fracture energy. The 

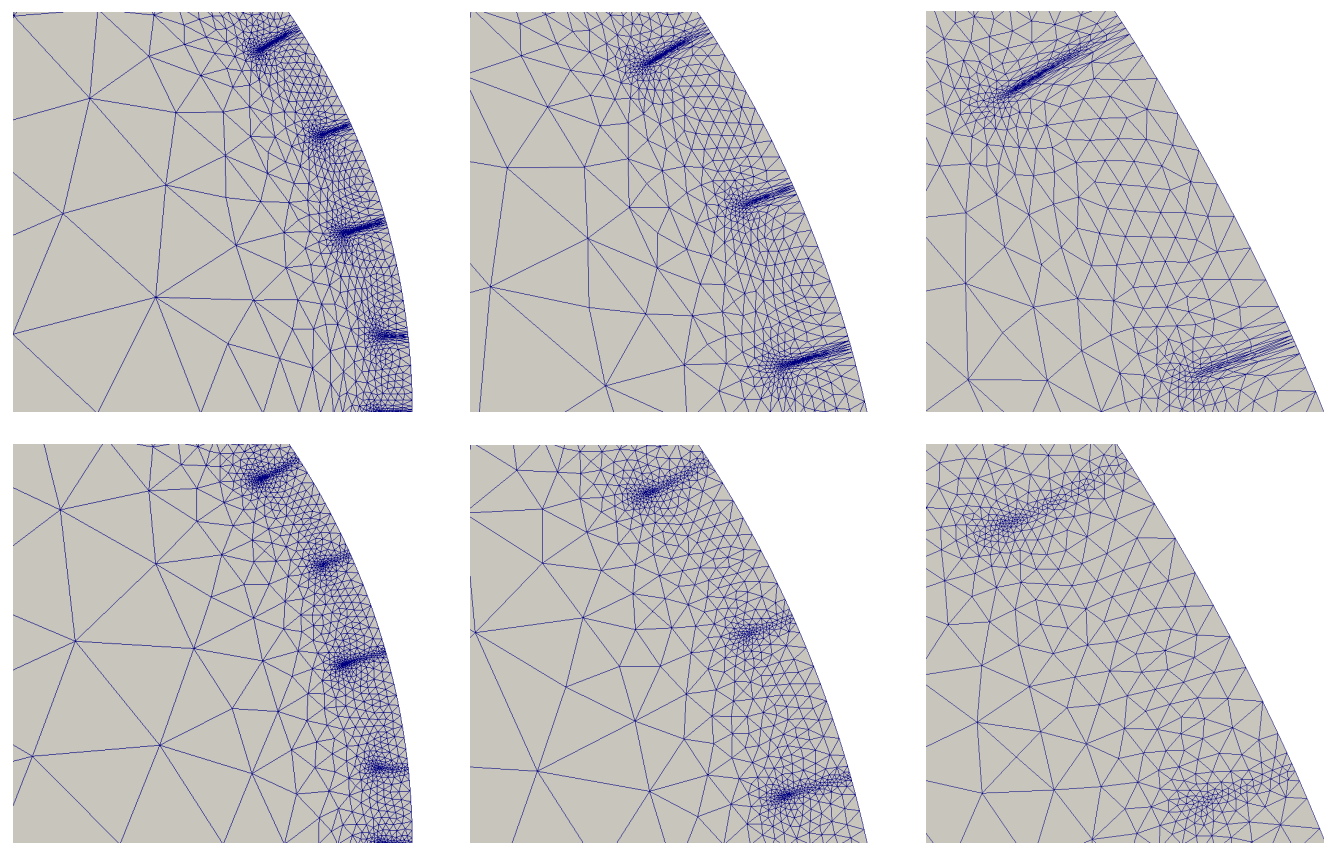

Figure 12: Disc brake: anisotropic (top) vs isotropic (bottom) mesh adaptation at time step 25, for increasing magnification (top-bottom).
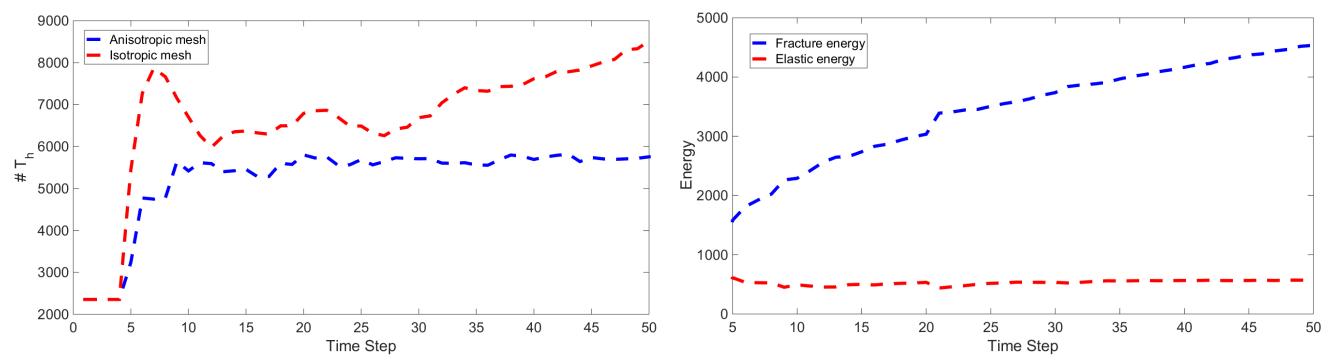

Figure 13: Disc brake: evolution of the mesh cardinality (left) and of the energy contributions (right).

trend confirms the constant increase of the fracture lengths throughout the time span.

\begin{tabular}{cccc}
\hline \hline & total time [s] & optimization time [s] & adaptation time [s] \\
\hline Anisotropic & 6342.62 & 4886.20 & 1456.42 \\
Isotropic & 9326.64 & 6794.20 & 2532.44 \\
\hline
\end{tabular}

Table 3: Disc brake: total, optimization, and adaptation time for the anisotropic and isotropic mesh adaptation. 

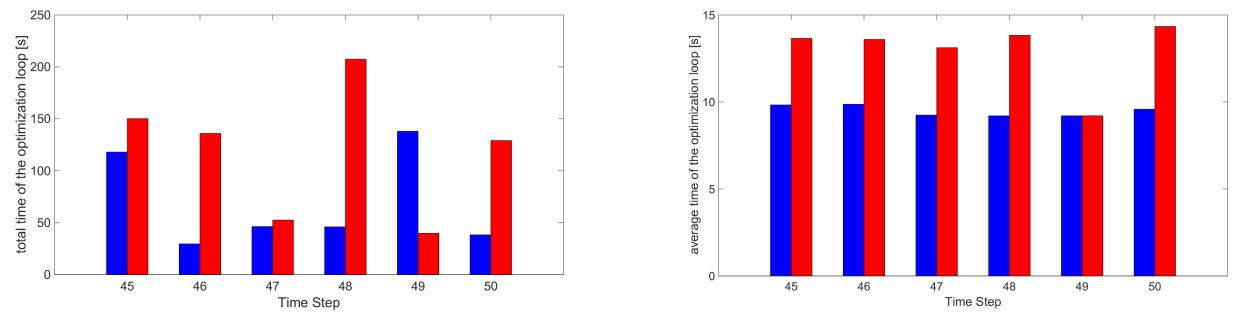

Figure 14: Disc brake: total and average time for the optimization loop at the last six time steps, for the anisotropic (left bars) and isotropic (right bars) procedures.
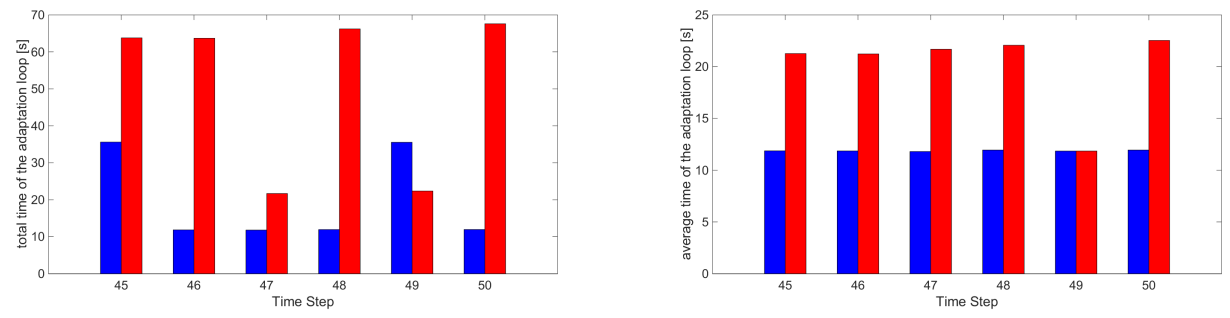

Figure 15: Disc brake: total and average time for the adaptation at the last six time steps, for the anisotropic (left bars) and isotropic (right bars) procedures.

\section{Conclusions}

We have extended the anisotropic approach provided in $[6,7,8]$ for the anti-plane and plane elasticity where crack genesis is biased by suitably notching the specimen and crack propagation is driven by an applied external displacement field, to the more challenging framework where both crack ignitation and propagation are driven by a thermal shock, which depends on the solution of the heat equation. Overall, we are dealing with a one-way coupled thermo-mechanical model, where only the mechanical part depends on the thermal setting, but not vice versa. From a theoretical viewpoint, we have derived an anisotropic a posteriori estimator to control the discretization error on the total energy. This theoretical tool supports the MACProX algorithm which alternates optimization and mesh adaptation to track the evolution of the damage field in an efficient way. The numerical assessment confirms the good performance of this algorithm on two reference test cases, with respect to a corresponding isotropic procedure. In future developments, we shall be concerned with the parallelization of the numerical code with a view to 3D applications and, possibly, with a comparison with actual experimental tests.

\section{Acknowledgements}

Part of this work was supported by the GNCS 2017 Project Advanced numerical methods combined with computational reduction techniques for parameterised PDEs and applications. 


\section{References}

[1] B. Bourdin, J.-J. Marigo, C. Maurini, P. Sicsic, Morphogenesis and propagation of complex cracks induced by thermal shocks, Phys. Rev. Lett. 112 (1) (2014) 014301-1-014301-5.

[2] J.-J. Marigo, C. Maurini, P. Sicsic, Initiation of a periodic array of cracks in the thermal shock problem: a gradient damage modeling, J. Mech. Phys. Solids 63 (2013) 256-284.

[3] H. A. Bahr, G. Fischer, H. J. Weiss, Thermal-shock crack patterns explained by single and multiple crack propagation, J. Mater. Sci. 21 (1986) 2716-2720.

[4] J. Geyer, S. Nemat-Nasser, Experimental investigation of thermally induced interacting cracks in brittle solids, Int. J. Solid. Struct. 18 (4) (1982) 349-356.

[5] J. Bisschop, F. K. Wittel, Contraction gradient induced microcracking in hardened cement paste, Cement Concr. Compos. 33 (4) (2011) 466-473.

[6] M. Artina, M. Fornasier, S. Micheletti, S. Perotto, Anisotropic mesh adaptation for crack detection in brittle materials, SIAM J. Sci. Comput. 37 (4) (2015) B633-B659.

[7] M. Artina, M. Fornasier, S. Micheletti, S. Perotto, Anisotropic adaptive meshes for brittle fractures: parameter sensitivity, in: A. Abdulle, S. Deparis, D. Kressner, F. Nobile, M. Picasso (Eds.), In Numerical Mathematics and Advanced Applications. Series: Lect. Notes Comput. Sci. Eng., Vol. 103, ENUMATH 2013, the 10th European Conference on Numerical Mathematics and Advanced Applications, Springer, 2015, pp. 293-302.

[8] M. Artina, M. Fornasier, S. Micheletti, S. Perotto, The benefits of anisotropic mesh adaptation for brittle fractures under plane-strain conditions, in: S. Perotto, L. Formaggia (Eds.), In New Challenges in Grid Generation and Adaptivity for Scientific Computing, Vol. 5 of SEMA SIMAI Springer, Springer Cham, 2015, pp. 43-67.

[9] L. Ambrosio, V. M. Tortorelli, Approximation of functionals depending on jumps by elliptic functionals via $\gamma$ convergence, Comm. Pure Appl. Math. 43 (8) (1990) 999-1036.

[10] L. Ambrosio, V. M. Tortorelli, On the approximation of free discontinuity problems, Boll. Unione Mat. Ital. 6 (4) (1992) 105-123.

[11] M.-A. Keip, B. Kiefer, J. Schröder, C. Linder, (Editors), Phase field approaches to fracture, Comput. Methods Appl. Mech. Engrg. 312 (2016) 1-634.

[12] L. Formaggia, S. Micheletti, S. Perotto, Anisotropic mesh adaption with application to CFD problems, in: H. Mang, F. Rammerstorfer, J. Eberhardsteiner (Eds.), Proceedings of WCCM V, Fifth World Congress on Computational Mechanics, 2002, (CD-ROM Edition).

[13] S. Micheletti, S. Perotto, Reliability and efficiency of an anisotropic Zienkiewicz - Zhu error estimator, Comput. Methods Appl. Mech. Engrg. 195 (9-12) (2006) 799-835.

[14] L. Dedè, S. Micheletti, S. Perotto, Anisotropic error control for environmental applications, Appl. Numer. Math. 58 (2008) 1320-1339.

[15] S. Micheletti, S. Perotto, Output functional control for nonlinear equations driven by anisotropic mesh adaption: the Navier-Stokes equations, SIAM J. Sci. Comput. 30 (6) (2008) 2817-2854.

[16] S. Micheletti, S. Perotto, The effect of anisotropic mesh adaptation on PDE-constrained optimal control problems, SIAM J. Control Optim. 49 (4) (2011) 1793-1828.

[17] R. Becker, R. Rannacher, An optimal control approach to a posteriori error estimation in finite element methods, Acta Numer. 10 (2001) 1-102.

[18] S. Burke, C. Ortner, E. Süli, An adaptive finite element approximation of a variational model of brittle fracture, SIAM J. Numer. Anal. 48 (2010) 980-1012.

[19] A. A. Griffith, The phenomena of rupture and flow in solids, Philosophical Transactions of the Royal Society of London A: Mathematical, Physical and Engineering Sciences 221 (582-593) (1921) 163-198.

[20] B. Bourdin, J.-J. Marigo, G. Francfort, The variational approach to fracture, J. Elast. 91 (1-3) (2008) 5-148

[21] J.-J. Marigo, G. Francfort, Revisiting brittle fracture as an energy minimization problem, J. Mech. Phys. Solids 46 (8) (1998) 131-1342.

[22] M. Hofacker, C. Miehe, A phase field model of dynamic fracture: Robust field updates for the analysis of complex crack patterns, Int. J. Numer. Methods Engng 93 (3) (2013) 276-301.

[23] C. Miehe, M. Hofacker, F. Welschinger, A phase field model for rate-independent crack propagation: Robust algorithmic implementation based on operator splits, Comput. Methods Appl. Mech. Engrg. 199 (45-48) (2000) 2756-2778.

[24] C. Kuhn, R. Müller, A continuum phase field model for fracture, Eng. Fract. Mech. 77 (18) (2010) 3625-3634.

[25] M. J. Borden, C. V. Verhoosel, M. A. Scott, T. J. R. Hughes, C. M. Landis, A phase-field description of dynamic brittle fracture, Comput. Methods Appl. Mech. Engrg. 217-220 (2012) 77-95.

[26] S. Burke, C. Ortner, E. Süli, An adaptive finite element approximation of a generalized Ambrosio-Tortorelli functional, Math. Models Methods Appl. Sci. 23 (9) (2013) 1663-1697.

[27] N. Kikuchi, J. T. Oden, Contact Problems in Elasticity: A Study of Variational Inequalities and Finite Element Methods, Vol. 8 of SIAM Studies in Applied Mathematics, 1988. 
[28] P. G. Ciarlet, The Finite Element Method for Elliptic Problems, North-Holland, Amsterdam, 1978.

[29] J. Brandts, S. Korotov, M. Křížek, The discrete maximum principle for linear simplicial finite element approximations of a reaction-diffusion problem, Linear Algebra Appl. 429 (2008) 2344-2357.

[30] P. Clément, Approximation by finite element functions using local regularization, RAIRO Anal. Numér. 2 (1975) $77-84$.

[31] L. Formaggia, S. Perotto, New anisotropic a priori error estimates, Numer. Math. 89 (4) (2001) 641-667.

[32] L. Formaggia, S. Perotto, Anisotropic error estimates for elliptic problems, Numer. Math. 94 (2003) 67-92.

[33] J. Z. Zienkiewicz, O. C. Zhu, The superconvergent patch recovery and a posteriori error estimates. Part 1: The recovery technique, Int. J. Numer. Methods Engng 33 (1992) 1331-1364.

[34] R. Rodríguez, Some remarks on Zienkiewicz-Zhu estimator, Numer. Methods Partial Differential Equations 10 (5) (1994) 625-635.

[35] F. Hecth, New developement in Freefem++, J. Numer. Math. 20 (3-4) (2012) 251-265.

[36] B. Bourdin, G. A. Francfort, J.-J. Marigo, Numerical experiments in revisited brittle fracture, J. Mech. Phys. Solids 48 (2000) 797-826.

[37] A. Wächter, L. T. Biegler, On the implementation of an interior-point filter line-search algorithm for large-scale nonlinear programming, Math. Program., Ser. A 106 (2006) 25-57. 


\section{MOX Technical Reports, last issues}

\section{Dipartimento di Matematica \\ Politecnico di Milano, Via Bonardi 9 - 20133 Milano (Italy)}

09/2017 Antonietti, P.F.; Ferroni, A.; Mazzieri, I.; Paolucci, R.; Quarteroni, A.; Smerzini, C.; Stupazzin Numerical modeling of seismic waves by Discontinuous Spectral Element methods

10/2017 Pini, A.; Stamm, A.; Vantini, S.

Hotelling's $\$ T^{\wedge} 2 \$$ in separable Hilbert spaces

08/2017 Ambrosi, D.; Beloussov, L.V.; Ciarletta, P.

Mechanobiology and morphogenesis in living matter: a survey

06/2017 Ekin, T.; Ieva, F.; Ruggeri, F.; Soyer, R.

On the Use of the Concentration Function in Medical Fraud Assessment

07/2017 Cabassi A.; Pigoli D.; Secchi P.; Carter P.A.

Permutation tests for the equality of covariance operators of functional data with applications to evolutionary biology

05/2017 Menafoglio, A.; Hron, K.; Filzmoser, P.

Logratio approach to distributional modeling

04/2017 Dede', L; Garcke, H.; Lam K.F.

A Hele-Shaw-Cahn-Hilliard model for incompressible two-phase flows with different densities

02/2017 Arena, M.; Calissano, A.; Vantini, S.

Monitoring Rare Categories in Sentiment and Opinion Analysis - Expo

Milano 2015 on Twitter Platform.

03/2017 Fumagalli, I.; Parolini, N.; Verani, M.

On a free-surface problem with moving contact line: from variational principles to stable numerical approximations

01/2017 Riccobelli, D.; Ciarletta, P.

Rayleigh-Taylor instability in soft elastic layers 